\title{
Spreading in narrow channels
}

\author{
C. Dotti, ${ }^{1,2}$ A. Gambassi, ${ }^{1,2}$ M. N. Popescu, ${ }^{1,2}$ and S. Dietrich ${ }^{1,2}$ \\ ${ }^{1}$ Max-Planck-Institut für Metallforschung, Heisenbergstr. 3, D-70569 Stuttgart, Germany \\ ${ }^{2}$ Institut für Theoretische und Angewandte Physik, \\ Universität Stuttgart, Pfaffenwaldring 57, D-70569 Stuttgart, Germany
}

\begin{abstract}
We study a lattice model for the spreading of fluid films, which are a few molecular layers thick, in narrow channels with inert lateral walls. We focus on systems connected to two particle reservoirs at different chemical potentials, considering an attractive substrate potential at the bottom, confining side walls, and hard-core repulsive fluid-fluid interactions. Using kinetic Monte Carlo simulations we find a diffusive behavior. The corresponding diffusion coefficient depends on the density and is bounded from below by the free one-dimensional diffusion coefficient, valid for an inert bottom wall. These numerical results are rationalized within the corresponding continuum limit.

PACS numbers: 02.50.-r, 05.70.Ln, 68.15.+e, 81.15.Aa
\end{abstract}

\section{INTRODUCTION}

In recent years, substantial progress has been made in the development of the "lab on a chip" concept, i.e., the integration of many physical and chemical processes (e.g., transport through micro-channels, mixing of different fluids, chemical reactions) into a single device; entire laboratory setups, like a gas chromatograph, have been miniaturized on a single chip (for a review see, e.g., Ref. 1]). In this context, microfluidics is becoming a standard tool in many applications, ranging from biology (see, e.g., Ref. 2]) to the handling of toxic or rare substances. Further scaling down to nanofluidics is expected to take place in the future [3]. Already now it is possible to sculpture channels with lateral dimensions of few tens of nanometers [4] (for a review on such fabrication processes see Ref. [5]) and carbon nanotubes have been proposed as possible pipes in nanofluidics [6, 7]. Chemically patterned substrates have also been suggested as a solution for directed transport, gating, mixing, or separation of fluids at the micro- and nano-scale [8]. In this case the channel consists of a strip of wettable material embedded in a non-wettable substrate so that the fluid flows along the wettable region and is laterally confined by the chemical contrast.

If one of the dimensions of a fluid film is comparable to the size of the fluid molecules, a hydrodynamical description of the film is no longer justified [9, 10, 11]. In this case the discrete nature of the fluid becomes important and the fluid cannot be treated as a continuum in the confined direction. In order to investigate such systems one possible approach is to carry out computer simulation of discrete models, e.g., molecular dynamics, kinetic Monte Carlo (KMC), or lattice Boltzmann simulations; recent work in this direction includes fluids in carbon nanotubes [6] and on chemically patterned substrates [12].

With the scaling down of microfluidic devices one has to deal with and may exploit the ultrathin precursor film which spreads ahead of the bulk fluid. Experimental studies have shown that in some cases such precursor films have molecular thickness 13, 14, 15, 16,
17, 18, 19, 20]. The spreading of such monolayers has been studied using a two-dimensional lattice gas Ising model [10, 21, 22] in which a half-space is occupied by a particle reservoir. Recent KMC simulations and a continuum analysis 23] of that model provided results in good qualitative agreement with available experimental data, and a further extension to the case of chemically patterned substrate has been proposed [24].

Fluids in narrow channels have been investigated theoretically in the context of single-file diffusion, i.e., when fluid particles cannot overtake each other (see, e.g., Refs. 25, 26, 27, 28, 29, 30, 31, 32, 33, 34]). Such systems show the interesting feature of non-diffusive behavior of tracer particles, which stimulated experimental (see, e.g., Refs. [33, 34]) and numerical [27, 29, 30, 31] interest. Here we present a lattice model for ultrathin films in which multiple occupancy of a site is allowed (generalizing the single-occupancy model of Refs. [10, 21, 22]) and in which the substrate-particle attractive interaction is decaying as a power law, whereas the particle-particle interaction is assumed to be hard-core repulsive only. This mimics the case in which the fluid-substrate interaction strongly dominates over the actual attractive long-range part of the fluid-fluid interaction. Based on the phenomena occurring in this minimalistic model, the extension to the case in which the attractive part of the fluid-fluid interaction is relevant will be presented elsewhere. We shall restrict our analysis to a one-dimensional model, which can effectively describe fluids in extremely narrow channels with a width which is less than twice the particle diameter. The sidewalls act to confine the particles. The corrugation of the substrate potential both at the bottom and at the sides is incorporated effectively by considering a lattice model for the particles. Due to the small thickness of the channel the transversal variation of the substrate potential can be ignored. This model is supposed to mimic not only molecular fluids but also colloidal particles in solution, with the colloidal particle setting the length scale.

We discuss both the initial dynamics, in which a fluid film fed by a reservoir gradually fills the channel, and the steady state, in which the fluid film in the channel is in 
contact with two reservoirs at different chemical potentials.

The paper is organized such that in Sec. II we define the model whereas in Sec. III the results of our Monte Carlo simulations are presented. The analyses of the diffusion-like dynamics and of the steady-state properties are presented in Sec. [V] In Sec. $\nabla$ we discuss the mean-field continuum limit of the model and rationalize, within this approximation, the results for the diffusion coefficient presented in Sec. IV] Section VI summarizes the main findings and provides our conclusions.

\section{THE MODEL}

Before specifying the rules defining the model, we further describe the general physical picture of the type of systems we have in mind.

As stated above, the fluid is assumed to be confined to a narrow, effectively one-dimensional channel. The sidewalls are very high compared with the fluid particle diameter, so that the fluid cannot spill out of the channel. The channel walls act on the particles such that only the vertical variation of the substrate potential matters. The left and the right end of the channel are connected to a feeding and absorbing particle reservoir, respectively, and the channel is initially empty. The fluid film inside the channel is taken to be compact, i.e., molecules are densely packed to form vertical columns without vacancies. This corresponds to the case in which the substrate is strongly attractive and vacancies inside columns are eliminated on a time scale much shorter than the typical time for exchanges of particles between columns. We describe these exchanges in terms of rates, which are related to the change of the energy of the system due to the corresponding move. Particle exchanges between columns and particle insertions and removals near the reservoirs are the only processes we consider. We assume that neither evaporation nor condensation takes place inside the channel. This minimalistic model aims at identifying general aspects and the main qualitative features of fluids spreading in a strongly confined geometry, rather than providing an accurate description of a particular physical situation.

Inspired by this picture, in Subsec. II we specify the configuration space and the corresponding energy function. In Subsec. IIB the dynamical rules (i.e., the allowed changes of the configurations and the associated rates) governing the time evolution in the bulk are discussed, whereas Subsec. IIC deals with the definition of the dynamics at the feeding and absorbing boundaries.

\section{A. Configurations and Hamiltonian}

The model is defined on a one-dimensional $(D=1)$ lattice, with sites $[0, \ldots,(L+1) a]$. The distance $a$ between two consecutive sites is assumed to be equal to the

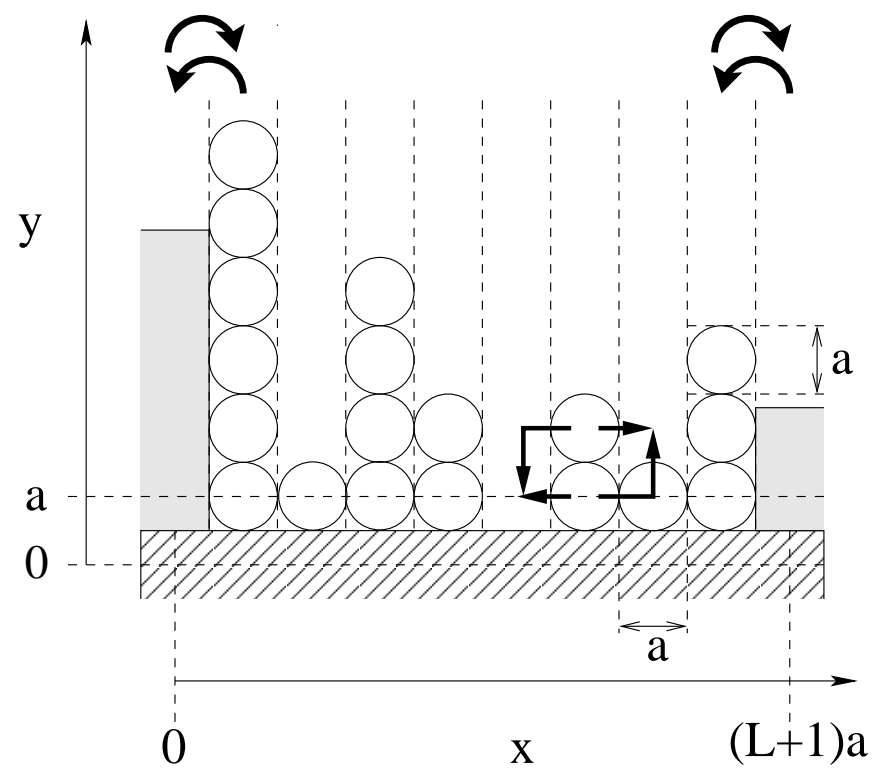

FIG. 1: A typical configuration of the model. The possible moves in the bulk are indicated by straight arrows, while the curved arrows denote reservoirs-system exchanges. The substrate, including the exclusion zone of its top layer, corresponds to the hatched area. The grey areas at $x \leq 0$ and $x \geq(L+1) a$ indicate reservoirs and the fluid particles are shown as circles.

effective diameter of a fluid particle, which is set by the hard-core repulsion between the particles. In the following, the site indices and the distances will be expressed in units of $a$. The two sites indexed with 0 and $L+1$ are the boundaries of the left and right particle reservoirs, respectively. The other sites, $[1, \ldots, L]$ (called 'bulk' in the following), represent the channel of length $L$ (assumed to be long, i.e., $L \gg 1$ ).

At every site $x \in[0, L+1]$ an occupation number $n_{x} \in \mathbb{N}_{0}$ specifies the number of particles piled in the column $x$ (see Fig. 1). Within the column, particles centers are located at integer $y$ positions. Accounting for fluid-substrate hard-core repulsion we consider the position $y=0$ as passing through the centers of the particles forming the top layer of the substrate. If the diameter of the substrate particles differs from that of the fluid particles, one may introduce an extra parameter to characterize the position of the fluid-substrate contact layer; for simplicity, however, we assume that the fluid particles in the first layer are located at $y=1$ (see Fig. (1).

The substrate is assumed to be uniform, and, consistent with our one-dimensional model, two-dimensional semi-infinite in the $y<0$ - direction. We denote the (attractive) pair interaction between a substrate particle and a fluid particle by $U_{s f}^{(p)}$, resembling dispersion forces:

$$
U_{s f}^{(p)}(d)= \begin{cases}-\frac{w_{s f}}{d^{6}}, & \text { for } d \geq 1 \\ \infty, & \text { for } d<1\end{cases}
$$

where $d$ is the dimensionless distance in units of $a$ between the substrate particle located at $\left(x^{\prime},-y^{\prime} \leq 0\right)$, and 
the fluid particle located at $(1 \leq x \leq L, y \geq 1)$. In the case of pairwise additive interactions, for a semi-infinite substrate $\left(x^{\prime} \in \mathbb{R}, y^{\prime} \in \mathbb{R}_{+}\right)$and in the continuum limit $(d \gg 1)$, this leads to a total substrate potential

$$
U_{s f}(y)=-w_{s f} \int_{0}^{\infty} \mathrm{d} y^{\prime} \int_{-\infty}^{\infty} \mathrm{d} x^{\prime} \frac{1}{\left[\left(y^{\prime}+y\right)^{2}+\left(x-x^{\prime}\right)^{2}\right]^{3}},
$$

i.e.,

$$
U_{s f}(y)= \begin{cases}-\frac{w_{s p}^{\prime}}{y^{4}}, & \text { for } y \geq 1 \\ \infty, & \text { for } y<1\end{cases}
$$

where $w_{s f}^{\prime}=\frac{3 \pi}{32} w_{s f}$. Within this ansatz, the particlesubstrate interaction in Eq. (3) depends on the height $y$ of the particle only. The energy of the fluid configuration $\left\{n_{1}, \ldots, n_{L}\right\}$ exposed to the substrate potential $U_{s f}$ is thus given by

$$
H_{s f}=\sum_{x=1}^{L} \sum_{y_{x}=1}^{n_{x}} U_{s f}\left(y_{x}\right),
$$

where the inner sum is defined to be 0 if $n_{x}=0$. Note that, following the discussion at the beginning of the present section, we assume that columns are always densely packed, so that configurations are as depicted in Fig. 1] since the configurations are characterized completely by a succession of numbers $\left\{n_{1}, \ldots, n_{L}\right\}$, the energy is a function of these numbers only, as in Eq. (4).

The same form [Eq. (1)] of the pair potential is assumed for the fluid particle - fluid particle interaction, where the corresponding interaction strength is denoted by $w_{f f}$. Each pair of particles separated by a distance $d_{f f} \geq 1$ contributes to the particle-particle energy, so that the total energy due to particle-particle interactions can be written as

$$
\begin{aligned}
& H_{f f}=\frac{1}{2} \sum_{x=1}^{L} \sum_{x^{\prime}=1}^{L} \sum_{y_{x}=1}^{n_{x}} \sum_{y_{x^{\prime}}=1}^{n_{x^{\prime}}} \\
& U_{f f}^{(p)}\left(d_{f f}=\sqrt{\left(x-x^{\prime}\right)^{2}+\left(y_{x}-y_{x^{\prime}}\right)^{2}}\right),
\end{aligned}
$$

with $U_{f f}^{(p)}(0)=0$ and the sums over $y_{x}$ and $y_{x^{\prime}}$ are taken to be zero if $n_{x}=0$ or $n_{x^{\prime}}=0$. The total energy function is $H[C]=H_{f f}+H_{s f}$ where $C \equiv\left\{n_{1}, \ldots, n_{L}\right\}$ characterizes completely each configuration. Note that this part of the Hamiltonian is restricted to the bulk; in general the reservoir-bulk interactions should also be accounted for separately. In the special case of the absence of longrange particle-particle interaction, i.e., $w_{f f}=0$, both the bulk and the reservoir-bulk contributions vanish, and the energy is $H[C]=H_{s f}$. As mentioned in the Introduction, in the following we shall discuss only this situation; the case $w_{f f} \neq 0$ will be presented elsewhere.

\section{B. The rates and the dynamics}

In this subsection we define and discuss the rates which govern the stochastic dynamics in the bulk, i.e., for $x \in$ $[1, L]$. The dynamics at the boundaries, $x=0$ and $x=$ $L+1$, will be discussed in the following subsection.

We assume that each particle in the column $x$ may jump into one of the nearest neighbor $(\mathrm{NN})$ columns $x+1$ or $x-1$. We introduce the rate $r_{C C^{\prime}}\left(y, y^{\prime}\right)$, which is the rate for a particle in column $x$ and at given height $y$, to jump to the next column $x+1$ and at height $y^{\prime}$. Within our aforementioned model assumption this process involves an instantaneous column height reduction by one in column $x$ and a column height increase in column $x+1$. This also means that the jumping particle is considered to be able to squeeze into column $x+1$ at position $y^{\prime}$ by pushing the particles above this position up by one unit; on the other hand if $y^{\prime}$ is above the top particle of column $x+1$, it falls down in order to form again a compact column. Accordingly the configurations $C, C^{\prime}$,

$$
\begin{aligned}
C & =\left\{n_{1}, \ldots, n_{x}, n_{x+1}, \ldots, n_{L}\right\} \\
C^{\prime} & =\left\{n_{1}, \ldots, n_{x}-1, n_{x+1}+1, \ldots, n_{L}\right\},
\end{aligned}
$$

represent the initial and the final configurations, for any pair $y, y^{\prime}$. Analogous considerations can be carried out for moves from $x+1$ to $x$, where the above configurations are interchanged. Therefore, within our model, the rates $r_{C C^{\prime}}\left(y, y^{\prime}\right)$ depend only on the initial and final configurations $C$ and $C^{\prime}$, respectively. Accordingly, the dependence on $y, y^{\prime}$ is dropped. We introduce the dimensionless rate $\tilde{u}_{C C^{\prime}}$, which is also assumed to depend only on $C, C^{\prime}$,

$$
\tilde{u}_{C C^{\prime}}=\frac{r_{C C^{\prime}}}{\nu_{0}},
$$

where $\nu_{0}$ fixes the time-scale of the model and we assume it to be independent of the source and target columns filling, i.e., the same for any particle in the source column. ( $\nu_{0}$ can be interpreted as the rate for a particle to jump to the NN column if the energy happens to be unchanged by the move.) In the following, times are measured in units of $\nu_{0}$, i.e., the dimensionless simulation time $t$ corresponds to an actual time $t_{a}=t / \nu_{0}$.

We choose the rates $\tilde{u}$ for all possible moves from column $x$ to column $x+1$ such that detailed balance

$$
\frac{\tilde{u}_{C C^{\prime}}}{\tilde{u}_{C^{\prime} C}}=\mathrm{e}^{-\beta \Delta H\left[C, C^{\prime}\right]}
$$

is obeyed, where $\Delta H\left[C, C^{\prime}\right]=H\left[C^{\prime}\right]-H[C]$ is the energy difference between the final $\left(C^{\prime}\right)$ and the initial $(C)$ configuration. Detailed balance has been chosen in order to ensure that thermal equilibrium is reached in the longtime limit, if the two reservoirs of particles at the right and the left of the channel are set to the same chemical potential. A possible choice that satisfies the detailed balance condition is

$$
\tilde{u}_{C C^{\prime}}=\mathrm{e}^{-\frac{\beta}{2} \Delta H\left[C, C^{\prime}\right]} .
$$


The chosen form of the rates [Eq. (9)] includes both "slow" $(\Delta H>0)$ and "fast" $(\Delta H<0)$ processes, and we implicitly assume that it captures essential features of the real dynamics.

The rate $\tilde{u}_{C C^{\prime}}$ is the same for any particle in the source column $x$, so that the total rate $u_{C C^{\prime}}$ for a column to decrease its occupation number by one, while a given NN column increases its own occupation number by one, is

$$
u_{C C^{\prime}}=n_{x} \tilde{u}_{C C^{\prime}}=n_{x} \mathrm{e}^{-\frac{\beta}{2} \Delta H\left[C, C^{\prime}\right]} .
$$

The rates in Eq. (10) are defined on the space of configurations specified by occupation numbers only. Detailed balance still holds and the corresponding Boltzmann statistical weight is

$$
p_{B}\left(n_{1}, \ldots, n_{x}, \ldots, n_{L}\right) \propto \frac{\mathrm{e}^{-\beta H}}{n_{1} ! \ldots n_{L} !}
$$

which accounts for "particle undistinctness" by dividing the Boltzmann factor by $n_{1} !, \ldots, n_{L}$ ! where $n_{x}$ ! is the number of choices to label the $n_{x}$ particles in each $x \in$ $[1, \ldots, L]$ column. In the case of the particle-substrate interaction described by the Hamiltonian in Eq. (4), the rate in Eq. (10) has the following explicit form:

$$
u\left(n_{x}, n_{x+1}\right)=n_{x} \exp \left\{\frac{\beta}{2}\left[\frac{w_{s p}^{\prime}}{\left(n_{x+1}+1\right)^{4}}-\frac{w_{s p}^{\prime}}{n_{x}^{4}}\right]\right\} .
$$

This formula emphasizes that $u$ depends only on the occupation numbers of the initial and the target column. The notation is such that the first argument stands for the source column (here located at $x$ with occupation $n_{x}$ ), while the second argument represents the target column (here at $x+1$ with occupation $n_{x+1}$ ).

Assuming that the dynamics leads to a diffusion-like behavior (as will be discussed in Sec. IV), some qualitative features of the diffusion coefficient as a function of the local density can be anticipated from the general properties of the rates in Eq. (12). First, consider the situation in which both $n_{x}=n$ and $n_{x \pm 1}=m$ are large compared to $\left(\beta w_{s p}^{\prime}\right)^{1 / 4}$. Then the exponent in Eq. (12) is very small and $u(n, m) \rightarrow n$, so that the model reduces to free particles diffusing in $D=1$. The same conclusion holds for $n=m+1$, in which case the exponent is zero leading to $u(n, m)=n$. In general, $u(n, m) \gtrless n$ if $n \gtrless m+1$; accordingly, jumps from high columns to low columns are faster than in the free case, while the opposite processes are slower. This means that diffusion, which tends to smooth out density gradients, is enhanced by the exponential factor in Eq. (12). Since at low densities most of the configurations are composed either of empty columns or of columns occupied by one particle, the most probable rate is $w(1,0)=1$, which results in free diffusion at low densities.

These considerations lead to the conclusion that the diffusion coefficient is expected to exhibit a peak at relatively low densities, because the rates exhibit the maximal difference with free diffusion rates if the target column is empty.
Before passing to the definition of the dynamics at the boundaries, we briefly comment on similar models which have been considered in the literature. In Refs. [35, 36] a class of dynamical models, to which our model belongs, is introduced and studied. In this class of models the rates depend on both the source and the target column, they do not necessarily satisfy detailed balance, and jumps occur not only between NN. (These models are known in the literature as misanthropic processes.) The main result of Refs. [35, 36] is that under certain conditions in the infinite square lattice it is possible to obtain an exact expression for the steady-state distribution. A concise summary of these results can be found in Refs. [37, 38], where their relevance for the non-equilibrium dynamics of interacting particles has been stressed. Applied to our case, the results in Refs. 35, 36] recover the equilibrium Boltzmann distribution in Eq. (11), with the Hamiltonian defined in Eq. (4), but do not provide information on the dynamics and steady-state distribution if chemical drive, caused by different chemical potential for the two reservoirs at the boundaries, is applied.

\section{Dynamics at the boundaries}

We consider now the dynamics at the boundaries and discuss two possible implementations. The first choice is to fix the occupation number of the columns 0 and $L+1$ at values $n_{0}$ and $n_{L+1}$, respectively, and to impose, with some additional assumptions, the same dynamics as in the "bulk". The boundary dynamics changes the occupation number $n_{1}$ of the first column of the system, according to Eq. (12), while the occupation number $n_{0}$ is unchanged by the move, and the same holds at $x=L$. One can physically motivate such a choice by assuming that the particle exchanges within the reservoir are so fast, so that a particle extracted from the reservoir is immediately replaced. Under this assumption the density of particles in the reservoir is simply $n_{0}$. While this choice seems to be rather natural, as explained in the following the equilibrium (i.e., for $n_{0}=n_{L+1}$ ) properties display an unexpected feature, i.e., a jump discontinuity in the density between the reservoirs and the system.

The total Hamiltonian in Eq. (4) is a sum of singlecolumn terms, so that the equilibrium grand canonical distribution factorizes:

$$
P_{e q}\left(\left\{n_{1}, \ldots, n_{L}\right\}\right)=\frac{1}{Z(w, \mu)} \prod_{k=1}^{L} p_{w}\left(n_{k}\right) \mathrm{e}^{-\mu n_{k}},
$$

where

$$
Z(w, \mu)=\left(\sum_{n=0}^{\infty} p_{w}(n) \mathrm{e}^{-\mu n}\right)^{L}
$$

is the total partition function, $\mu=\beta \tilde{\mu}$ is the dimensionless chemical potential, with $\tilde{\mu}$ as the actual chemical potential, $p_{w}$ is a non-normalized single-column statistical 
weight,

$$
p_{w}(m)=\frac{1}{m !} \exp [2 w h(m)]
$$

$w=\beta w_{s p}^{\prime} / 2$ is a dimensionless quantity, and

$$
h(m)= \begin{cases}\sum_{k=1}^{m} \frac{1}{k^{4}}, & \text { for } n \geq 1, \\ 0, & \text { for } n=0 .\end{cases}
$$

The chemical potential $\mu$ controls the mean density of particles in the system,

$$
\rho_{e q}(z) \equiv \frac{N_{e q}}{L}=\frac{z}{L} \partial_{z} \ln [Z(w, z)]=\frac{\sum_{n \geq 1} n p_{w}(n) z^{n}}{\sum_{n \geq 0} p(n) z^{n}},
$$

where $N_{e q}$ is the mean total number of particles in the system, and $z=\mathrm{e}^{-\mu}$ is the fugacity. Detailed balance for the rates at the left reservoir reads

$$
\begin{aligned}
& P_{e q}\left(\left\{n_{1}, \ldots, n_{L}\right\}\right) u\left(n_{0}, n_{1}\right)= \\
& =P_{e q}\left(\left\{n_{1}+1, \ldots, n_{L}\right\}\right) u\left(n_{1}+1, n_{0}\right)
\end{aligned}
$$

where the probability per unit time of inserting a particle into the column at $x=1$ is compared with the corresponding probability per unit time of removing the particle in the same column. A similar condition has to hold at the right end $x=L$ of the system. Combining Eq. (18) with Eqs. (12) and (13) leads to

$$
z=n_{0} \exp \left\{-w\left[\frac{1}{n_{0}^{4}}+\frac{1}{\left(n_{0}+1\right)^{4}}\right]\right\} .
$$

Equations (17) and (19) give the equilibrium density $\rho_{e q}$ in the system as a function of $n_{0}$. As expected, if $n_{0}$ is large $\rho_{\text {eq }}$ coincides with $n_{0}$ : in Eq. (19) $n_{0} \gg w^{1 / 4}$ implies $z \approx n_{0}$, and in Eq. (17) $n_{0} \gg w^{1 / 3}$ implies $p_{w}(n) \approx$ $\exp (2 w \zeta(4)) / n$ !, so that for $n_{0} \gg \max \left(w^{1 / 3}, w^{1 / 4}\right)$ Eq. (17) reduces to

$$
\rho_{e q} \approx \mathrm{e}^{-z} z \frac{\partial}{\partial z} \mathrm{e}^{z}=z \approx n_{0}, \quad n_{0} \gg \max \left(w^{1 / 3}, w^{1 / 4}\right) .
$$

In the range of substrate potential strength we investigated $(0.5<w<5)$ the approximation $\rho_{e q} \approx n_{0}$ is valid if $n_{0}>5$. As the substrate potential strength is increased, this threshold increases, while it tends to zero for $w \rightarrow 0$. For densities lower than the threshold, the reservoir occupation number $n_{0}$ does not coincide with the density $\rho_{e q}$ of the equilibrium system as obtained from Eqs. (13)-(17) and (19) (see Fig. 2). For example, in the case $n_{0}=3$ one has $\rho_{e q} \simeq 3.19$ for $w=2$ and $\rho_{e q} \simeq 3.23$ for $w=4$. These densities are in very good agreement with the simulation data for the density $\rho_{1}$ in the first column (see, c.f., Fig. [5(b)). In the simulations we investigated a non-equilibrium situation in which the

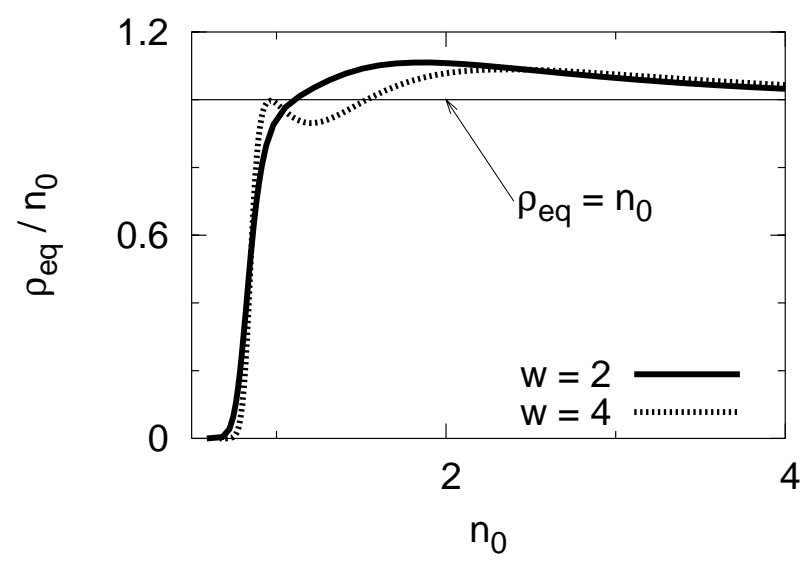

FIG. 2: The equilibrium density $\rho_{e q}$ [Eqs. (13)-(17) and (19)], divided by the reservoir occupation number $n_{0}$, as a function of $n_{0}$ for $w=2$ and $w=4$, respectively.

two reservoirs at the boundaries have different occupation numbers $\left(n_{0} \neq n_{L+1}\right)$; nevertheless we recover the equilibrium density in the first column. This shows how it is possible to control the densities at the first $(x=1)$ and last $(x=L)$ site by varying the occupation numbers $n_{0}$ and $n_{L+1}$. In order to obtain arbitrary densities, it is necessary to take $n_{0}$ and $n_{L}$ to be continuous, thus loosing the direct physical interpretation of these parameters. Moreover, as shown in Fig. 2, $\rho_{e q}$ drops sharply for $n_{0} \lesssim 0.8$, and in the range $0 \lesssim \rho_{e q} \lesssim 0.8$ a high numerical accuracy would be required to determine the corresponding value of $n_{0}$.

These two problems can be solved by generalizing the dynamics at the boundaries as follows. In Eq. (12) the terms depending on $n_{0}$ and $n_{L+1}$, i.e., the properties of the reservoirs, are replaced by constants $\alpha, \gamma, \delta$, and $\kappa$ in the following way:

$$
\begin{aligned}
& u_{\alpha}\left(n_{1}\right)=\alpha \exp \left[\frac{w}{\left(n_{1}+1\right)^{4}}\right], u_{\gamma}\left(n_{1}\right)=\gamma n_{1} \exp \left[-\frac{w}{n_{1}^{4}}\right], \\
& u_{\delta}\left(n_{L}\right)=\delta \exp \left[\frac{w}{\left(n_{L}+1\right)^{4}}\right], u_{\kappa}\left(n_{L}\right)=\kappa n_{L} \exp \left[-\frac{w}{n_{L}^{4}}\right],
\end{aligned}
$$

where $u_{\alpha}$ is the rate for particle insertion into column $n_{1}$ from the left reservoir, $u_{\gamma}$ is the rate for particle removal from column $n_{1}$ into the left reservoir and $u_{\delta}, u_{\kappa}$ are the corresponding rates at $x=L$. Imposing detailed balance [Eq. (18)] for the rates defined in Eq. (21) gives

$$
\mathrm{e}^{-\mu}=z=\frac{\alpha}{\gamma}=\frac{\delta}{\kappa} .
$$

In the non-equilibrium case the fugacity of the right reservoir, denoted as $z_{L+1}=\delta / \kappa$, and the one of the left reservoir, i.e., $z_{0}=\alpha / \gamma$, are different $\left(z_{0} \neq z_{L+1}\right)$. Using these two fugacities in Eq. (17), the densities of the two corresponding equilibrium systems are found; we $d e$ fine them to be the reservoir densities. In simulations 
we proceed backwards: first we choose $\rho_{0}=\rho_{e q}\left(z_{0}\right)$ and $\rho_{L+1}=\rho_{e q}\left(z_{L+1}\right)$, and then we find the corresponding ratios by inverting Eq. (17). Setting $\gamma=\kappa=1$ implies $\alpha=z_{0}, \delta=z_{L+1}$ so that the inversion is simpler.

\section{MONTE-CARLO SIMULATIONS}

The continuous time dynamics defined by the rules described in Subsecs. IIB and IIC is simulated using a Kinetic Monte Carlo (KMC) method [39]. At every step an increment $\Delta t$ for the time variable is drawn from the distribution

$$
\begin{aligned}
& P(\Delta t)=\frac{1}{S\left(n_{0}, \ldots, n_{L+1}\right)} \exp \left[S\left(n_{0}, \ldots, n_{L+1}\right) \Delta t\right], \\
& S\left(n_{0}, \ldots, n_{L+1}\right)=\sum_{x=0}^{L}\left[u\left(n_{x}, n_{x+1}\right)+u\left(n_{x+1}, n_{x}\right)\right],
\end{aligned}
$$

where $S$ is the total rate to leave the configuration $\left\{n_{0}, \ldots, n_{L+1}\right\}$. The move to perform is then chosen according to the weight $u / S$ of its rate. We used a classical $\mathrm{N}$-fold way algorithm [40], which has the advantage that the selected moves are accepted without rejection. The model depends on four parameters: the substrate interaction strength $w=w_{s p}^{\prime} \beta / 2$ in units of the thermal energy, the two boundary densities $n_{0}$ and $n_{L+1}$, and the length $L$ of the system. The simulations have been performed up to a maximum time $\tau_{\text {tot }}$ and quantities have been measured after the initial time $\tau_{0}$. The simulations covered both the spreading and the steady-state regime and in both cases we sampled the same set of quantities. In order to keep the notation simple we indicate averages always with $\langle\cdot\rangle$, but, as described in the following, the meaning of the symbol is different in the two situations considered.

The mean density of particles, i.e., the density at site $x$ and time $t$ is defined as

$$
\rho(x, t)=\left\langle n_{x}(t)\right\rangle,
$$

while the total mean number of particles (or mean total mass) is

$$
M(t)=\sum_{x=1}^{L}\left\langle n_{x}(t)\right\rangle .
$$

The transport properties have been studied using the integrated particle current at site $x$ and time $t$,

$$
J(x, t, \Delta t)=\Delta n_{x, x+1}(t, t+\Delta t)-\Delta n_{x+1, x}(t, t+\Delta t),
$$

where $\Delta n_{x, x^{\prime}}(t, t+\Delta t)$ is the number of particles jumping from site $x$ to site $x^{\prime}$ within the time interval $\Delta t$. We define a mean instantaneous current as

$$
\langle j(x, t)\rangle=\lim _{\Delta t \rightarrow 0} \frac{\langle J(x, t, \Delta t)\rangle}{\Delta t} .
$$

\section{A. Spreading}

For the spreading regime the right reservoir has been converted into a particle sink by setting $n_{L+1}=0$, while $n_{0}=11$ and $L=1000$. In the simulations performed with these values of the parameters the leakage of particles through the sink is negligible for times $t \lesssim 10^{4}$. We studied both the initial-time dynamics by setting $\tau_{0}=0$ and $\tau_{\text {tot }} \leq 10^{4}$ and the long-time behavior in which both reservoirs play a role (see, c.f., Fig $4, w=0.5$ ); in this latter case we have chosen $\tau_{\text {tot }}=5 \times 10^{4}$ and $\tau_{0}=10^{4}$ in order to reduce the CPU and memory requirements. In the spreading regime we implemented the simulation average by drawing different sequences of (pseudo-) random numbers while keeping the initial condition fixed so that in this case $\langle\cdot\rangle$ is the ensemble average; the typical number of runs we averaged over is 2000 . We studied the shape of the density profile $\rho(x, t)$, defined in Eq. (24), as a function of the interaction strength $w$ in the range $0.5 \lesssim w \lesssim 1.5$.

Since for $w=0$ the dynamics reduces to free diffusion, it is natural to check if in the general case (i.e., $w \neq 0$ ) the profiles show a diffusive scaling. Plotting them as a function of $\lambda=x / \sqrt{t}$ we indeed obtain a collapse of data measured at different times, as shown in Fig. 3. The rescaled profiles are similar to the one for free diffusion, except for a small bend at densities around 1 (see Fig 3(b)), which depends on the interaction strength $w$. The diffusive scaling is confirmed by the time evolution of the total mass [Eq. (25)] shown in Fig. (4) which is expected to evolve as $M \propto \sqrt{t}$. We observe deviations from this behavior only at short times, when the boundary dynamics dominates, and at long times, when the leakage of particles through the sink becomes relevant.

\section{B. Steady state}

The steady state is reached after running the simulation for an initial thermalization time $\tau_{0}\left(\tau_{0} \approx 10^{5}\right.$ for $L=1000$, chosen by checking that for $t>\tau_{0}$ the observables are time independent) and saving the configurations generated every sampling time interval $\tau_{s}$, with $\tau_{s}=200$ for $L=1000$. The average $\langle\cdot\rangle$ for the observables defined above is taken over this set of configurations. The choice for $\tau_{s}$ is a compromise between speed and having as small correlations between the $N_{s}$ measurements as possible. We assume that the total simulation time $\tau_{\text {tot }}=N_{s} \tau_{s}+\tau_{0}$ is sufficient to explore a significant part of the phase space, so that the performed average coincides with the average over the (unknown) steady state distribution. Note that this assumption is justified because no signs of dynamical phase transitions (which would introduce extremely long time scales) are found in the simulations.

The density $\rho(x)$ [Eq. (24)] exhibits a profile smoothly interpolating between the two reservoirs (see Fig. (5) and slightly deviating from the corresponding free diffusion 

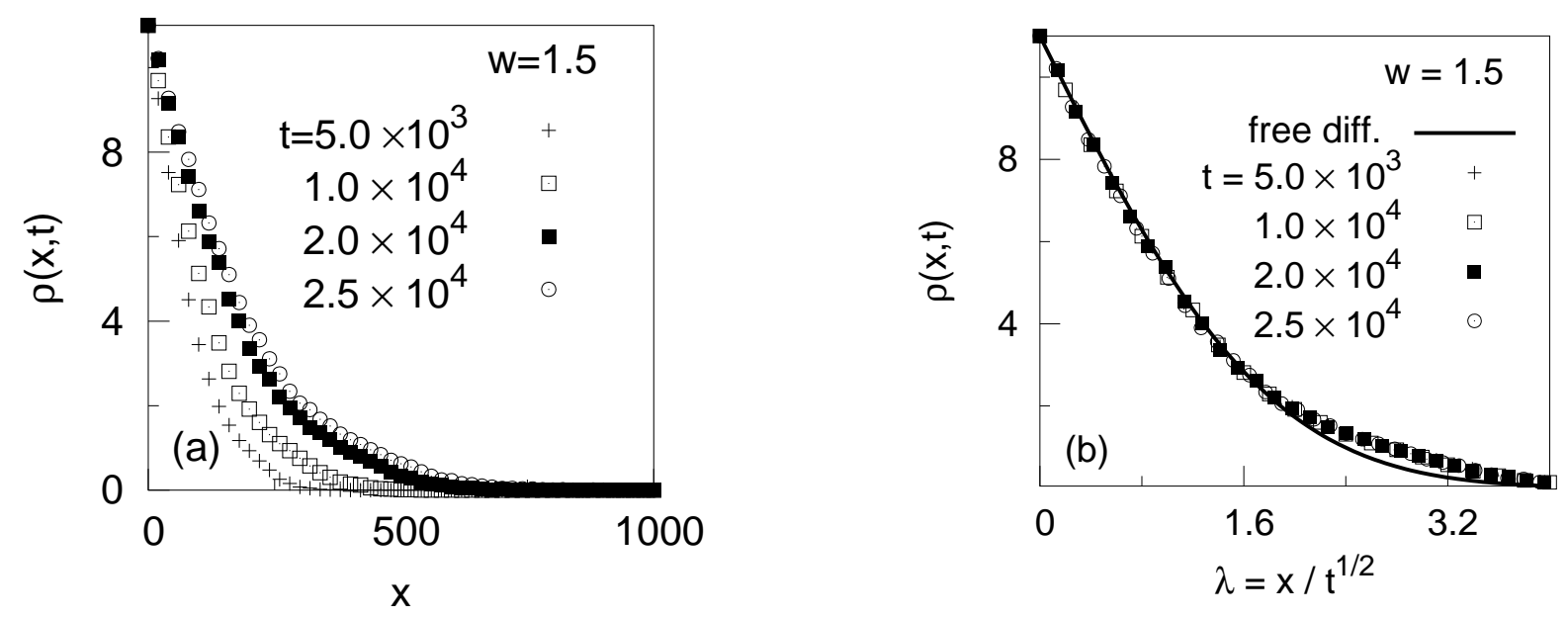

FIG. 3: Time-dependent density profiles for spreading in a system with $L=1000$, for $n_{0}=11, n_{L+1}=0$, and $w=1.5$ at times $t=5 \times 10^{3}(+), 10^{4}(\square), 2 \times 10^{4}(\boldsymbol{\square})$, and $2.5 \times 10^{4}(\odot)$ as a function of $x(\mathrm{a})$, and of $\lambda=x / \sqrt{t}(\mathrm{~b})$, respectively. The solid line indicates the scaling function for free diffusion (i.e., $w=0$ ).

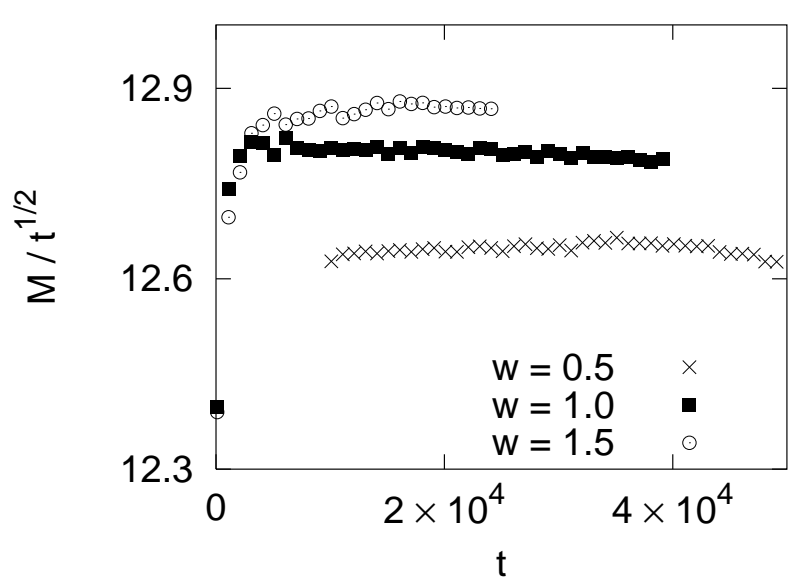

FIG. 4: Total mass $M / \sqrt{t}$ for spreading as a function of time $t$ for different values of the interaction $w$ with the substrate: $w=0.5$ (total simulation time $\tau_{\text {tot }}=5 \times 10^{4}$ and initial sampling time $\left.\tau_{0}=10^{4}\right)(\times), w=1.00\left(\tau_{t o t}=4 \times 10^{4}\right.$, $\left.\tau_{0}=0\right)(\boldsymbol{\square})$, and $w=1.50\left(\tau_{\text {tot }}=2.5 \times 10^{4}, \tau_{0}=0\right)(\odot)$ For all symbols $L=1000, n_{0}=11$, and $n_{L+1}=0$.

profile which is a straight line. This deviation is considered in more detail in Appendix B, where its dependence on both the interaction and the reservoirs densities is analyzed. In the steady state both reservoirs play a role and finite-size effects have to be checked; it turns out that for $L>200$ there is no detectable dependence of the data on the particular value of $L$ other than a trivial rescaling of the density profile.

We have also determined the current $\langle j\rangle$ defined in Eq. (27): in the steady state $\langle j\rangle$ does not depend on $t$, so that $J(x, t, \Delta t)=\langle j\rangle \Delta t$ for any sufficiently large time interval $\Delta t$. $J$ can be obtained by measuring the flux of particles between any two sites $x$ and $x+1$, because in the steady state the current $\langle j\rangle$ does not depend on $x$ due to local particle conservation. The instantaneous steady state current $\langle j(x)\rangle$ (a dependence on $x$ is indicated to recall the random fluctuations around the mean value $\langle j\rangle)$ can then be obtained from

$$
\langle j(x)\rangle=\frac{J\left(x, 0, \tau_{t o t}\right)-J\left(x, 0, \tau_{0}\right)}{N_{s} \tau_{s}} .
$$

Note that in Eq. (28) the current integrated over the thermalization time, $J\left(x, 0, \tau_{0}\right)$, which depends on $x$ and $t$, has been subtracted. We have calculated $\langle j(x)\rangle$ for $x \in[1, \ldots, L]$ leading to $\langle j\rangle=L^{-1} \sum_{x=1}^{L}\langle j(x)\rangle$.

\section{ANALYSIS OF THE SIMULATION DATA}

\section{A. Methods to determine the diffusion coefficient}

Guided by the results of the KMC simulations of the microscopic model we expect that a continuum (in space and time) description for the behavior of the model at long times and large spatial scales is possible, i.e., that the hydrodynamic limit exists and is well defined. A rigorous proof has been provided for a small number of models (see, e.g., Ref. [41]). In the present case such an explicit derivation appears to be a difficult task.

Assuming that the particle density $\rho$ and the current $\langle j\rangle$, defined in Eqs. (24) and (27), are smooth functions of the position $x$ and of the time $t$, the local conservation of particle density in the bulk (which is implicit in the dynamics of the model) is expected to take the form of a continuity equation:

$$
\partial_{t} \rho(x, t)=-\partial_{x}\langle j(x, t)\rangle .
$$

The results of the simulations strongly indicate a diffusive scaling at long times and large spatial scales, i.e., 

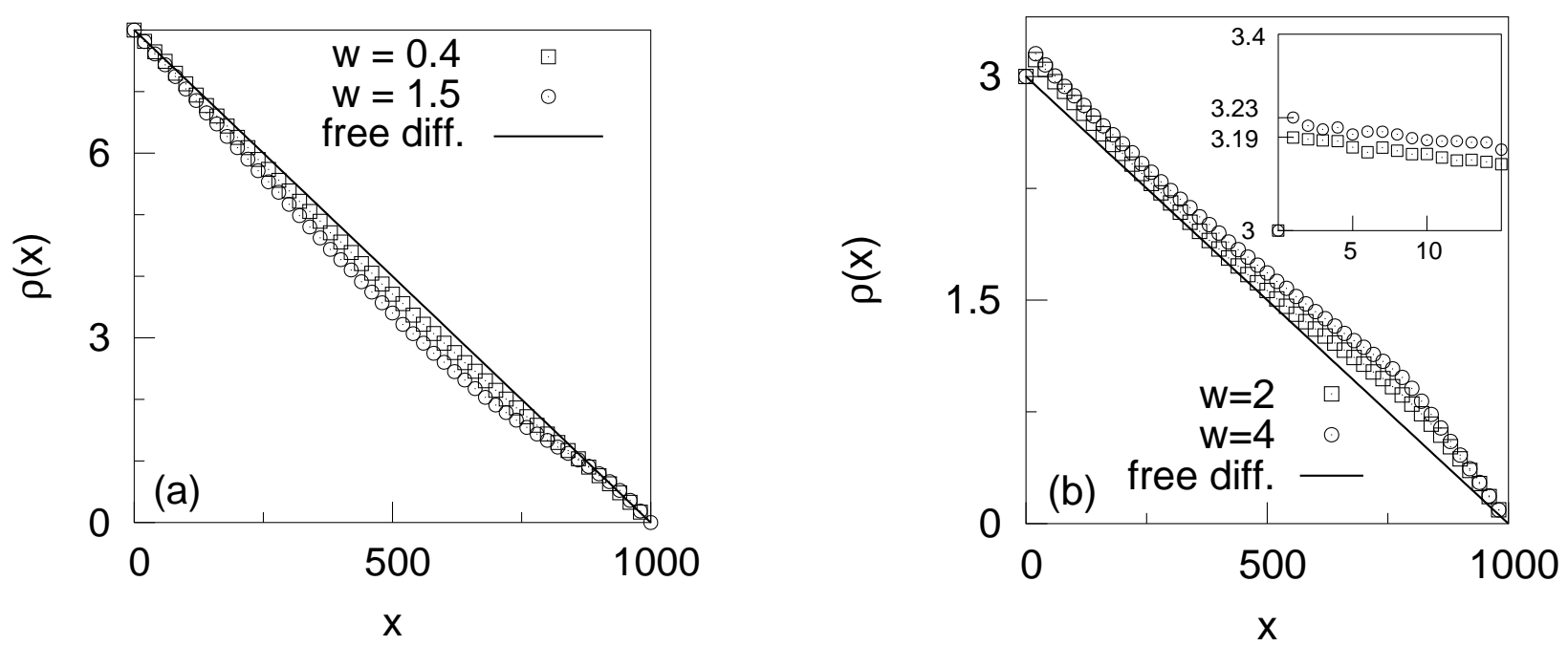

FIG. 5: Steady-state density profiles in a system of length $L=1000$ for (a) $w=0.4(\bullet)$ and $w=1.5(\odot), n_{0}=8, n_{L+1}=0$; (b) $w=2(\bullet)$ and $w=4(\odot), n_{0}=3, n_{L+1}=0$. The inset in (b) is a close-up view of the vicinity of the left reservoir showing $\rho_{e q} \simeq \rho_{1}>n_{0}\left[\rho_{e q} \simeq 3.19\right.$ for $w=2$ and $\rho_{e q} \simeq 3.23$ for $w=4$; see Eqs. (17) and (19) and the discussion in the main text]. In both (a) and (b) the free diffusion is indicated by a full line.

$\rho(x, t)=\bar{\rho}(x / \sqrt{t})$, suggesting that the dynamics amounts to non-linear diffusion:

$$
\begin{gathered}
\langle j(x, t)\rangle=-D(\rho) \partial_{x} \rho(x, t) \Rightarrow \\
\partial_{t} \rho(x, t)=\partial_{x}\left[D(\rho) \partial_{x} \rho(x, t)\right] .
\end{gathered}
$$

Based on the density profile $\rho(x, t)$ from the simulations, it is possible to extract the function $D(\rho)$ from the data in the spreading and the steady state regime, respectively, as follows.

- Spreading. Using the scaling behavior $\rho(x, t)=$ $\bar{\rho}(\lambda=x / \sqrt{t})$ (see the results in Sec. III), Eq. (30) reduces to

$$
\lambda \frac{\mathrm{d}}{\mathrm{d} \lambda} \bar{\rho}(\lambda)=\frac{\mathrm{d}}{\mathrm{d} \lambda}\left[D(\bar{\rho}(\lambda)) \frac{\mathrm{d}}{\mathrm{d} \lambda} \bar{\rho}(\lambda)\right] .
$$

Assuming that $\frac{\mathrm{d} \bar{\rho}}{\mathrm{d} \lambda} D(\rho \overline{(\lambda)})$ and $\bar{\rho}(\lambda)$ vanish for $\lambda \rightarrow$ $\infty$, which is supported by the simulation data $(L \gg 1$ is an approximation for $L \rightarrow \infty)$, integrating Eq. (31) and inverting $\bar{\rho}(\lambda)$ into $\lambda(\bar{\rho})$, one finds

$$
D(\bar{\rho})=\frac{\mathrm{d}}{\mathrm{d} \bar{\rho}} \lambda(\bar{\rho}) \int_{0}^{\bar{\rho}} \mathrm{d} \rho \lambda(\rho) .
$$

This method might be inaccurate for small densities due to a systematic effect. For $x \approx L$ the profile bends in order to fulfill the condition $n_{L+1}=0$, so that its derivative $\frac{\mathrm{d}}{\mathrm{d} \lambda} \bar{\rho}$ is larger than the derivative of a profile $\bar{\rho}_{\infty}$ in the infinite lattice: $\frac{\mathrm{d}}{\mathrm{d} \lambda} \bar{\rho}_{\infty}<\frac{\mathrm{d}}{\mathrm{d} \lambda} \bar{\rho}$. The integral in Eq. (32) is also underestimated, since $\lambda(\rho \rightarrow 0) \nrightarrow \infty$. These effects lead to an underestimated diffusion coefficient for small values of $\rho$. The most severe effect is probably due to the derivative $\frac{\mathrm{d}}{\mathrm{d} \lambda} \bar{\rho}(\lambda)$, while the errors in the integral can be partially corrected by using larger lattice sizes.

- Steady state. In the stationary state the current and the density are constant with respect to time, so that Eq. (30) leads to

$$
D(\rho)=-\frac{\langle j\rangle}{\rho^{\prime}},
$$

where $\rho^{\prime}(x)=\frac{\mathrm{d}}{\mathrm{d} x} \rho(x)$. Note that the computation of $D$ in this regime does not require any assumptions on $\rho$ and $\rho^{\prime}$, as in the previous case, and therefore no systematic deviations from the actual diffusion coefficient are expected.

- Steady state in quasi-equilibrium. A steady state deviating only slightly from the equilibrium is realized by imposing reservoir densities which differ only slightly. Equation (33) leads to

$$
\langle j\rangle=\frac{1}{L} \int_{\rho_{L}}^{\rho_{1}} \mathrm{~d} \rho D(\rho),
$$

where $\rho_{1}$ and $\rho_{L}$ are the densities at the first and the last site, respectively, and $L$ is the length of the system. For $\left|\rho_{1}-\rho_{L}\right| \ll \rho_{1}$ one has

$$
D\left(\frac{\rho_{1}+\rho_{L}}{2}\right) \approx \frac{L\langle j\rangle}{\left(\rho_{1}-\rho_{L}\right)}
$$



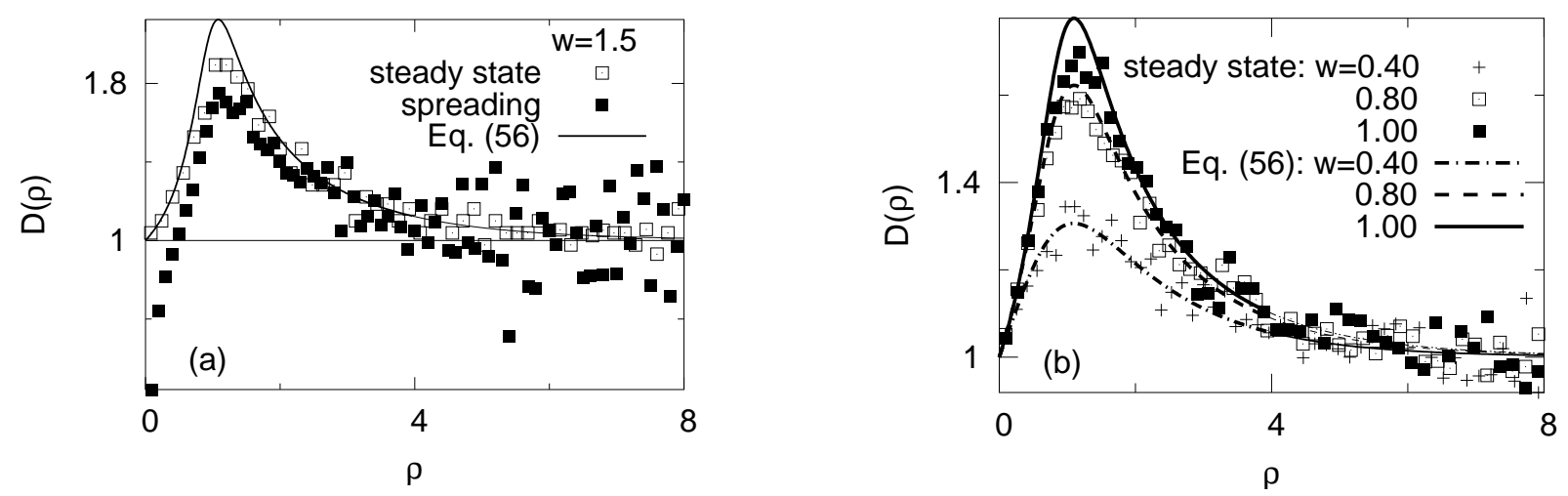

FIG. 6: (a) Nonlinear diffusion coefficient $D$ as a function of the density, obtained from the simulation data in the spreading regime $(\mathbf{\square})$ and in the steady state $(\square)$, as well as the corresponding analytical result [full line, Eq. ([56)]. All the data correspond to $w=1.5$ and $L=1000$.

(b) Nonlinear diffusion coefficient [Eq. (33)] from steady-state simulation data for $w=0.40(+), w=0.80(\square)$, and $w=1.00$

(ם) $(L=1000)$. Analytical results (lines) for $D(\rho)$ are calculated from Eq. (56).

\section{B. Results from the spreading regime}

In order to extract $D(\rho)$ from the numerical data for given $w$ and $L$, we have considered all the profiles in the scaling regime. We have binned the $\rho$ axis with $\Delta \rho=0.05$, averaged all the values $\lambda$ in each bin, and evaluated the function $\lambda(\rho)$ by interpolation of the resulting data points, while $\frac{\mathrm{d}}{\mathrm{d} \lambda} \bar{\rho}(\lambda)$ has been obtained by finite differences. The results for $D(\rho)$ obtained by using Eq. (32) are shown in Fig. 6(a). While it appears that, for large values of $\rho, D(\rho) \rightarrow 1$ as expected from the corresponding discussion in Subsec. IIB, for $\rho \rightarrow 0$ the diffusion coefficient goes to zero due to the systematic error in the derivative $\frac{\mathrm{d}}{\mathrm{d} \lambda} \bar{\rho}(\lambda)$, as explained in Subsec. IVA. The noise at large values of $\rho$ is due to determining the derivatives numerically, because for large $\rho$ the spatial fluctuations of the density are stronger.

The diffusion coefficient is peaked and the substrate potential enhances diffusion (see Subsec. IIB). The position of the peak $(\rho \simeq 1)$ cannot be predicted by qualitative arguments, but is in the range of low densities, as expected from the discussion in Subsec. IIB.

\section{Results from steady-state and quasi-equilibrium regimes}

In order to obtain $D(\rho)$ from the steady-state data by using Eq. (33) we have measured the average current and the average density profile. The latter has been appropriately binned (the density is averaged over 5 sites), in order to be able to evaluate the derivative via finite differences. The corresponding results are shown in Figs. 6(a), 6(b), and 7 We note that $D(\rho \rightarrow 0) \rightarrow 1$. The correct behavior at low densities is captured, while for large $\rho$ the data are still rather noisy, because the method to extract $D$ relies on determining derivatives numerically. The overall agreement between results ob- tained from the spreading data and from the steady-state data is good for $\rho \gtrsim 0.5$ and even better for $\rho \gtrsim 2$, which shows that the diffusion picture described in Subsec.IVA leads to consistent results.

Simulations under quasi-equilibrium conditions have been restricted to the case $w=2$ because the quantitative agreement between the results obtained from quasiequilibrium, using Eq. (35) for $D(\rho)$, and those obtained in the steady state is satisfactory (see Fig. 7). Due to the small difference in density of the two reservoirs $(\delta \rho=0.01)$ the average current is very small and requires accurate measurements. The data are obtained by averaging over $10^{7}$ configurations or more (approximately 100 times more than for the steady state data), leading to a high precision for the density profile, too. The autocorrelation time for the average density has been carefully checked and the time intervals between samples have been chosen in order to minimize correlations. The procedure allows one to estimate reliably the statistical error for the diffusion coefficient, shown by the errorbars in Fig. [7(a). Note that the results obtained from steady-state simulations with bigger reservoir differences lie within the errorbars.

\section{CONTINUUM DESCRIPTION}

In this section we derive the nonlinear diffusion equation corresponding to the continuum limit of the model. The equation is derived from the microscopic dynamics by using several simplifying assumptions. We start from the master equation, which describes exactly the dynamics of the model and in its most general form can be written as

$$
\partial_{t} P_{t}(C)=\sum_{C^{\prime}} \mathcal{M}\left(C, C^{\prime}\right) P_{t}\left(C^{\prime}\right)
$$

where $C$ is a generic configuration, while $\mathcal{M}\left(C, C^{\prime}\right)$ encodes the transitions from the configuration $C$ to the con- 

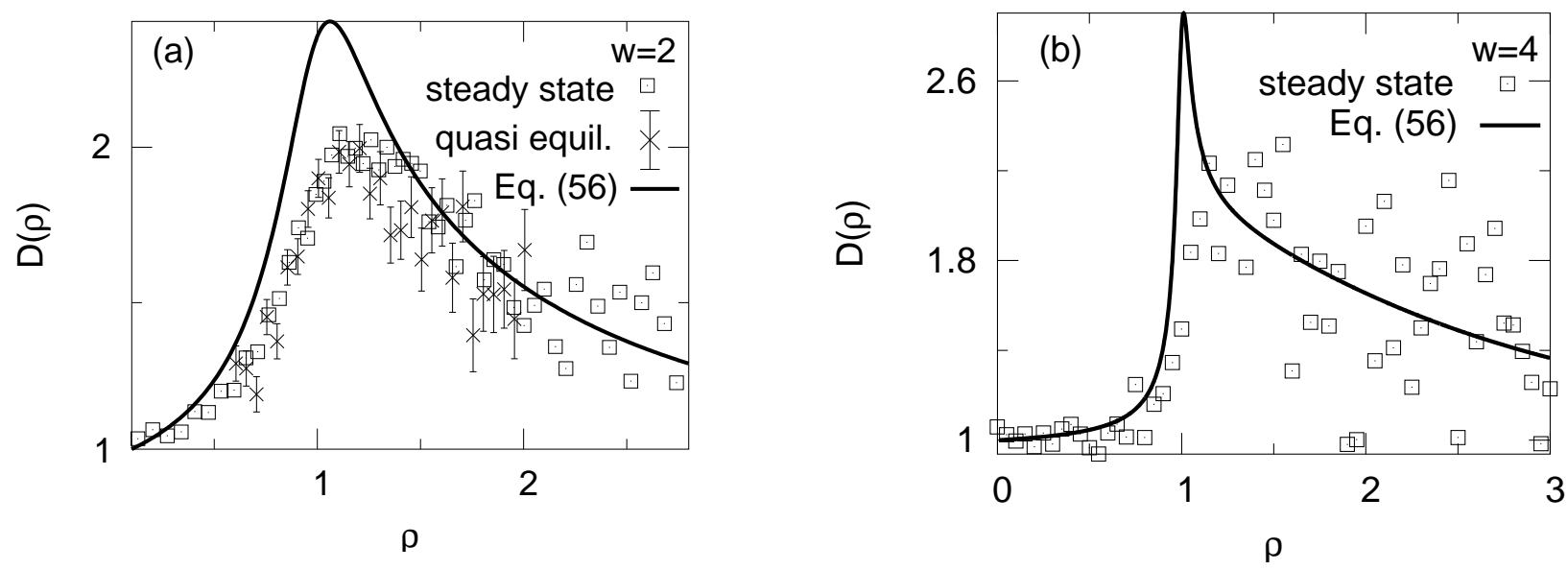

FIG. 7: Nonlinear diffusion coefficient $D(\rho)$ for large values of $w$. The open squares $(\square)$ are obtained from simulation data in the steady-state regime [Eq. (33)] for $w=2$ (a) and $w=4(\mathrm{~b})(L=1000)$. The lines correspond to analytical calculations [Eq. (56)]. The crosses $(\times)$ with errorbars in (a) are obtained from simulation data under quasi-equilibrium conditions [Eq. (35)].

figuration $C^{\prime}$. In our case, the operator $\mathcal{M}$ can be split into bulk $\left(\mathcal{M}_{b}\right)$ and boundary $\left(\mathcal{M}_{s}\right)$ terms, so that

$$
\partial_{t} P_{t}(C)=\sum_{C^{\prime}}\left[\mathcal{M}_{s}\left(C, C^{\prime}\right)+\mathcal{M}_{b}\left(C, C^{\prime}\right)\right] P_{t}\left(C^{\prime}\right)
$$

The operator $\mathcal{M}_{b}$ describes bulk moves, upon which particles are exchanged between columns at sites $x \in$ $[1, \ldots, L]$ and which are associated with the rates defined in Eq. (12). The operator $\mathcal{M}_{s}$, describes boundary moves upon which particles are inserted into or removed from the system at the sites $x=1$ or $x=L$, and which are associated with the rates introduced in Subsec. IIC Explicit expressions for the operators $\mathcal{M}_{b}$ and $\mathcal{M}_{s}$ are given in Appendix $\mathrm{A}$

The evolution of the ensemble average of a generic (time-indipendent) operator $\mathcal{O}$ can be obtained from Eq. (36) as

$$
\partial_{t}\langle\mathcal{O}\rangle=\sum_{C, C^{\prime}} \mathcal{O}(C) \mathcal{M}\left(C, C^{\prime}\right) P_{t}\left(C^{\prime}\right)
$$

where $\mathcal{O}(C)$ is the value of the operator $\mathcal{O}$ for configuration $C$. Recalling that $\mathcal{M}(C, C)=-\sum_{C^{\prime} \neq C} \mathcal{M}\left(C^{\prime}, C\right)$, it is straightforward to obtain

$$
\partial_{t}\langle\mathcal{O}\rangle=\sum_{C} \mathcal{K}(C) P(C)
$$

where $\mathcal{K}(C)$ is the jump moment of the operator $\mathcal{O}$ defined as

$$
\mathcal{K}(C) \equiv \sum_{C^{\prime} \neq C}\left[\mathcal{O}\left(C^{\prime}\right)-\mathcal{O}(C)\right] \mathcal{M}\left(C^{\prime}, C\right) .
$$

We consider now the operator $\mathcal{N}_{x}$, defined as $\mathcal{N}_{x}(C)=$ $n_{x}$. Accordingly, the configurations $C^{\prime}$, for which the jump moments defined in Eq. (40) are nonzero, are $C^{\prime}=$ $\left\{n_{1}, \ldots, n_{x-1}+1, n_{x}-1, n_{x+1}, \ldots, n_{L}\right\},\left\{n_{1}, \ldots, n_{x-1}-\right.$ $\left.1, n_{x}, n_{x+1}+1, \ldots, n_{L}\right\},\left\{n_{1}, \ldots, n_{x-1}, n_{x}+1, n_{x+1}-\right.$ $\left.1, \ldots, n_{L}\right\},\left\{n_{1}, \ldots, n_{x-1}, n_{x}-1, n_{x+1}+1, \ldots, n_{L}\right\}$ with $x \in[2, \ldots, L-1]$, so that using Eqs. (39), (40), (A1), and (A2) one obtains in the bulk

$$
\partial_{t} \rho(x, t)=-[\langle j(x+1, t)-j(x, t)\rangle],
$$

where $\rho(x, t)=\left\langle n_{x}\right\rangle, x \in[2, \ldots, L-1]$, and

$$
\langle j(x, t)\rangle=\left\langle u\left(n_{x-1}, n_{x}\right)-u\left(n_{x}, n_{x-1}\right)\right\rangle
$$

is the mean local and instantaneous current in Eq. (27). Assuming that the probability distribution [Eq. (37)] factorizes completely, i.e., within the mean field approximation

$$
P(C, t)=\prod_{y=1}^{L} \tilde{p}_{y}\left(n_{y}, t\right)
$$

the average rates in Eq. (42) [for the definition of the rates see Eq. (12)] reduce to

$$
\begin{aligned}
& \left\langle u\left(n_{x}, n_{x+1}\right)\right\rangle \equiv \sum_{n_{1}=1}^{\infty} \cdots \sum_{n_{L}=1}^{\infty} \\
& \prod_{y=1}^{L} \tilde{p}_{y}\left(n_{y}, t\right)\left\{n_{x} \exp \left[\frac{w}{\left(n_{x+1}+1\right)^{4}}-\frac{w}{n_{x}^{4}}\right]\right\} \\
& =\tilde{f}_{1}(w, t, x) \tilde{f}_{2}(w, t, x+1),
\end{aligned}
$$

where

$$
\tilde{f}_{1}(w, t, x)=\sum_{n_{x}=1}^{\infty} \tilde{p}_{x}\left(n_{x}, t\right) n_{x} \exp \left(\frac{w}{n_{x}^{4}}\right)
$$

and

$$
\tilde{f}_{2}(w, t, x)=\sum_{n_{x}=0}^{\infty} \tilde{p}_{x}\left(n_{x}, t\right) \exp \left[-\frac{w}{\left(n_{x}+1\right)^{4}}\right] .
$$


The second equation in Eq. (44) holds because the sum in the first line equals 1 for any $y \neq x, x+1$ due to the normalization of the distribution $\tilde{p}$. We further assume that the distribution $\tilde{p}_{x}$ depends smoothly on $x$ and $t$, and that it does so only via the mean site density $\rho(x, t)$ (or, equivalently, on an effective local chemical potential) such that $\tilde{p}_{x}(n, t)=p(n, \rho(x, t))$ and $\tilde{f}_{1,2}(w, t, x)=f_{1,2}(w, \rho(x, t))$. Expanding the density up to second order in $a$ [i.e., $\rho(x+a) \approx \rho(x)+a \partial_{x} \rho+\frac{1}{2} a^{2} \partial_{x}^{2} \rho$ ] and then setting $a=1$ [Eqs. (41) and (44)], leads to the diffusion equation

$$
\begin{aligned}
\partial_{t} \rho(x, t)= & {\left[f_{1}(w, \rho) \partial_{\rho} f_{2}(w, \rho)-f_{2}(w, \rho) \partial_{\rho} f_{1}(w, \rho)\right] } \\
& \times \partial_{x}^{2} \rho(x, t)
\end{aligned}
$$

with a density-dependent diffusion coefficient

$$
D(w, \rho)=f_{1}(w, \rho) \partial_{\rho} f_{2}(w, \rho)-f_{2}(w, \rho) \partial_{\rho} f_{1}(w, \rho) .
$$

In the steady-state regime the functions $f_{1,2}$ can be computed explicitly in the local equilibrium approximation, i.e., by approximating the exact steady-state distribution with a grand canonical equilibrium Gibbs distribution $P_{G}$ :

$$
P_{G}\left(n, w, \mu_{x}\right)=\frac{1}{Z\left(w, \mu_{x}\right)} \frac{1}{n !} \exp \left(2 w h(n)-\mu_{x} n\right)
$$

where

$$
Z\left(w, \mu_{x}\right)=\sum_{n=0}^{\infty} \frac{1}{n !} \exp \left(2 w h(n)-\mu_{x} n\right)
$$

$h$ is defined in Eq. (16) and, as before, $\mu_{x}=\beta \tilde{\mu}_{x}$ is a dimensionless chemical potential. These approximations are expected to hold if the density varies slowly in space, so that the parts of the system to the left and to the right of $x$ act on the column at $x$ effectively as a particle reservoir with a well-defined chemical potential $\mu_{x}$. Accordingly, a nontrivial profile $\rho_{x}$ emerges which smoothly interpolates between the reservoir densities at $x=0$ and $x=L+1$

The distribution $P_{G}$ can be expressed in terms of the local density $\rho_{x}$ by solving the implicit equation

$$
\left\langle n_{x}(w)\right\rangle=\sum_{n=1}^{\infty} P_{G}\left(n, w, \mu_{x}\right) n=\rho_{x}
$$

for $\mu_{x}=\mu\left(\rho_{x}\right)$. The functions $f_{1}$ and $f_{2}$ in Eq. (47) are then given by

$$
\begin{aligned}
& f_{1}(w, \rho)=\frac{1}{Z(w, \mu)} \sum_{n \geq 0} \\
& \left.\frac{1}{n !} \exp \left[2 w h(n)-\frac{w}{(n+1)^{4}}-\mu n\right]\right|_{\mu=\mu(\rho)},
\end{aligned}
$$

$$
\begin{aligned}
& f_{2}(w, \rho)=\frac{1}{Z(w, \mu)} \sum_{n \geq 1} \\
& \left.\frac{1}{(n-1) !} \exp \left(2 w h(n)+\frac{w}{n^{4}}-\mu n\right)\right|_{\mu=\mu(\rho)} .
\end{aligned}
$$

Due to Eq.(16) one has

$$
2 w h(n)+\frac{w}{(n+1)^{4}}=2 w h(n+1)-\frac{w}{(n+1)^{4}},
$$

which implies

$$
f_{2}=\mathrm{e}^{-\mu} f_{1}
$$

and thus

$$
\partial_{\mu} f_{2}=\mathrm{e}^{-\mu} \partial_{\mu} f_{1}-f_{2} .
$$

From Eq. (50) one can infer the derivative of $\rho$ with respect to $\mu$ :

$$
\begin{aligned}
\partial_{\mu} \rho & =\sum_{n=1}^{\infty} n \partial_{\mu} P_{G}=\sum_{n=1}^{\infty} n(\langle n\rangle-n) P_{G}= \\
& =\langle n\rangle^{2}-\left\langle n^{2}\right\rangle \equiv-\chi(w, \mu) .
\end{aligned}
$$

Combining Eqs. (47), (54), and (55) one obtains

$$
\begin{aligned}
D(w, \rho) & =\left.\frac{f_{1}(w, \mu) f_{2}(w, \mu)}{\chi(w, \mu)}\right|_{\mu=\mu(\rho)}= \\
& =\left.\frac{\left\langle u\left(n_{x}, n_{x+1}\right)\right\rangle}{\chi(w, \mu)}\right|_{\mu=\mu(\rho)} \cdot
\end{aligned}
$$

Equation (56) allows one to compute numerically the diffusion coefficient. To this end we solve Eq. (50) for $\mu(\rho)$ and insert the solution $\mu(\rho(x))$ back into Eq. (56). The resulting $D(\rho)$ is obtained by approximating the series in Eq. (51) by finite sums. We have checked the stability of the calculation for densities $0<\rho<11$, in order to be able to make contact with our Monte Carlo data. For $w<1.5$ the theoretical expression is in good agreement with the simulation data (see Fig. 6), but for larger values of the interaction significant deviations occur (see Fig. 7). These deviations systematically increase with increasing interaction strength, which cannot be easily blamed on numerical inaccuracies. A first possible explanation for the deviations could be the non-equilibrium character of the simulations, due to the chemical potential gradient present in the system. However, the quasiequilibrium simulation results allow us to rule out strong non-equilibrium effects as the primary source for these deviations, because $D(\rho)$ computed from these data coincides with the one obtained from non-equilibrium simulations; thus, the diffusion coefficient is basically independent of the difference between the reservoirs densities. Taking advantage of this fact we can make use of general results for an infinitely large system, derived in quasi-equilibrium conditions, such as the Green-Kubo 
formula [42]:

$$
\begin{aligned}
D(\rho)=\frac{1}{\chi} & {\left[\left\langle u\left(n_{x}, n_{x+1}\right)\right\rangle_{e q}\right.} \\
& \left.-\sum_{x^{\prime}=1}^{\infty} \int_{0}^{\infty} \mathrm{d} t^{\prime}\left\langle j_{x+1} U_{e q}\left(t^{\prime}\right) j_{x^{\prime}}\right\rangle_{e q}\right]
\end{aligned}
$$

where $\langle\cdot\rangle_{e q}$ indicates the average performed over the equilibrium distribution and $U_{e q}$ is the evolution operator for the equilibrium dynamics. Following the discussion in Ref. [42], in Appendix $\mathrm{A}$ we present a brief derivation of Eq. (57) for the class of models we are interested in. The comparison of Eqs. (56) and (57) shows that our meanfield calculation reproduces the first term in Eq. (57), while the terms which would reduce (in the appropriate limit) to the time integral of the current correlations cannot be captured by this mean-field approximation.

Computing explicitly the current correlations is difficult, but some of their general properties [42] allow us to conclude that they provide a qualitatively correct correction to the diffusion coefficient calculated via Eq. (56). The function $\left\langle j_{x+1} U_{e q}(t) j_{x^{\prime}}\right\rangle_{e q}$ appearing in Eq. (57) is integrable, positive, and decaying exponentially for $t \rightarrow \infty$. Thus it is a negative contribution to Eq. (57) and decreases the diffusion coefficient obtained from Eq. (56). This is in qualitative agreement with the data shown in Fig. 7.

\section{SUMMARY AND CONCLUSIONS}

We have introduced a lattice model (Fig. 1) for spreading of a fluid in narrow, quasi one-dimensional slit-like channels and in contact with particle reservoirs located at their ends. The model accounts for long-range attractive substrate-fluid interactions, while the fluid-fluid interaction is taken to be hard-core only. We have studied the spreading behavior and stationary state using kinetic Monte Carlo simulations and a non-linear diffusion equation corresponding to the continuum limit of our discrete model. The main results are the following.

The spreading regime has been studied starting from an empty lattice: we have set the right reservoir density to $\rho_{L+1}=0$, so that it acts as a perfect sink, and the left reservoir to a nonzero value (typically $\rho_{0}=11$ ), thereby feeding particles into the system. At intermediate times, for which the reservoirs do not play a relevant role, we have found a diffusion-like behavior, such that profiles at different times collapse onto a single master curve if the scaling variable $\lambda=x / \sqrt{t}$ is introduced (see Fig. 3). This scaling is further confirmed by the time evolution of the total mass [see Eq. (25)] shown in Fig. 4. In the steady state (Fig. 5) we have found nontrivial density profiles depending on the substrate interaction strength. The dynamics at the boundary influences the density profiles: the analysis in Subsec. IIC shows that one of the possible definitions of the boundary rates causes discontinuities in the profiles. These discontinuities can be eliminated by an alternative definition or can be calculated explicitly (see Fig. 2). The profiles also depend on the reservoir density as discussed in Appendix B, either lying completely above the free-diffusion straight line or crossing this line at a certain point. This feature can be described by the deviation $\Delta x(\rho)$ from free diffusion [see Eq. (B1) and Fig. 8 and explained in terms of the function $R(\rho)$ introduced in Eq. (B8). In Fig. 10 the general behavior of this function is sketched while in Fig. 9 the simulation and mean-field results for the deviation $\Delta x(\rho)$ are shown.

Assuming that a nonlinear diffusion equation describes the behavior of the particle density, we have extracted the diffusion coefficient from the simulation data in the spreading regime (Fig. 6(a)) and in the steady state (Figs. 6] and 7). The two sets of results are compatible with each other and show that the interaction with the substrate tends to enhance diffusion, as expected from qualitative arguments discussed in Subsec. IIB,

The Monte Carlo results for the diffusion coefficient are in agreement with an analytical calculation based on local equilibrium assumptions, in which the equilibrium grand canonical distribution is modified by a spatially varying local chemical potential. The agreement is very good for weak interactions with the substrate (Fig. 6), but deteriorates for strong interactions (Fig. 7). The independence of the diffusion coefficient from the difference between the reservoirs densities, suggested by quasi-equilibrium simulations, allows one to qualitatively explain these deviations by using a Green-Kubo formula.

An experimental situation to which our model would apply should be effectively quasi one-dimensional, with effective inert side walls confining the fluid and the substrate-fluid interaction strongly dominating over the fluid-fluid interaction. This might be realized, e.g., by colloidal particles in channels. An experimental investigation of the diffusion coefficient as a function of the density would allow one to make a direct comparison with the results presented here.

\section{APPENDIX A: A GREEN-KUBO FORMULA}

In this appendix we derive a Green-Kubo formula in the case of the non-equilibrium steady state induced by an infinitesimal difference $\delta_{\mu}=\mu_{0}-\mu_{L+1} \rightarrow 0$ between the dimensionless (in units of $k_{B} T$ ) chemical potentials $\mu_{0}$ and $\mu_{L+1}$ of the left and the right reservoir, respectively. The rates are denoted by $u\left(n_{x}, n_{x+1}\right)$ but they are not necessarily of the explicit form of Eq. (12). The master equation, which describes the dynamics of the system, is given by Eq. (37), where $\mathcal{M}_{b}$ is an operator that acts on a distribution $P$ as 


$$
\begin{aligned}
& \sum_{C^{\prime}} \mathcal{M}_{b}\left(C, C^{\prime}\right) P\left(C^{\prime}\right)=\sum_{x=1}^{L-1}\left\{-\left[u\left(n_{x}, n_{x+1}\right)+u\left(n_{x+1}, n_{x}\right)\right] P\left(n_{1}, \ldots, n_{L}\right)+\right. \\
& +u\left(n_{x+1}+1, n_{x}-1\right) P\left(n_{1}, \ldots, n_{x}-1, n_{x+1}+1, \ldots, n_{L}\right) \\
& \left.+u\left(n_{x}+1, n_{x+1}-1\right) P\left(n_{1}, \ldots, n_{x}+1, n_{x+1}-1, \ldots, n_{L}\right)\right\}
\end{aligned}
$$

and where $\mathcal{M}_{s}$ acts on $P$ as

$$
\begin{array}{r}
\mathcal{M}_{s}\left(C, C^{\prime}\right) P\left(C^{\prime}\right)=-\left[u\left(n_{1}, n_{0}\right)+u\left(n_{0}, n_{1}\right)+u\left(n_{L}, n_{L+1}\right)+u\left(n_{L+1}, n_{L}\right)\right] P\left(n_{1}, \ldots, n_{L}\right) \\
+u\left(n_{0}, n_{1}-1\right) P\left(n_{1}-1, \ldots, n_{L}\right)+u\left(n_{1}+1, n_{0}\right) P\left(n_{1}+1, \ldots, n_{L}\right) \\
+u\left(n_{L}+1, n_{L+1}\right) P\left(n_{1}, \ldots, n_{L}+1\right)+u\left(n_{L+1}, n_{L}-1\right) P\left(n_{1}, \ldots, n_{L}-1\right) .
\end{array}
$$

If the alternative definitions of the rates at the boundaries are employed [Eq. (21)] the rates $u_{\alpha, \kappa, \gamma, \delta}$ replace $u\left(n_{0}, n_{1}\right), u\left(n_{1}, n_{0}\right)$, and $u\left(n_{L}, n_{L+1}\right), u\left(n_{L+1}, n_{L}\right)$ in Eq. (A2).

We require that the rates $u$ satisfy detailed balance, so that for $\delta_{\mu}=0$, i.e., in the equilibrium case and thus without net transport of particles, the system is described by the grand canonical distribution

$$
P_{e q}^{G C}=P_{e q}^{C} \mathrm{e}^{-\mu_{0} N},
$$

where $P_{e q}^{C}$ is a canonical distribution and $N=\sum_{x=1}^{L} n_{x}$ is the total number of particles in the system. In the general case $\delta_{\mu} \neq 0$ detailed balance should be satisfied locally by the boundary rates at each end of the system.

The formal solution of the master equation (37) is

$$
P(t)=\mathrm{e}^{\mathcal{M} t} P_{\text {in }}=U(t) P_{\text {in }},
$$

where $P_{i n}$ is the initial distribution $P(t=0)$ and $U(t)=$ $\mathrm{e}^{\mathcal{M} t}$ is the time evolution operator. In the equilibrium case $\delta_{\mu}=0$ the corresponding dynamics is indicated by $\mathcal{M}_{\text {eq }}$. Splitting arbitrarily the operator $\mathcal{M}$ as $\mathcal{M}=\mathcal{M}_{0}+$ $\mathcal{M}_{I}$, one can check that from the equation

$$
U(t)=U_{0}(t)+\int_{0}^{t} \mathrm{~d} t^{\prime} U\left(t-t^{\prime}\right) \mathcal{M}_{I} U_{0}\left(t^{\prime}\right),
$$

where $U_{0}(t)=\mathrm{e}^{\mathcal{M}_{0} t}$, the two equations

$$
\begin{aligned}
& \partial_{t} U(t)=\mathcal{M} U(t) \\
& U(0)=1
\end{aligned}
$$

can be obtained. Since these equations have as unique solution the evolution operator $U(t)=\mathrm{e}^{\mathcal{M} t}$, Eq. A4) holds. Assuming that unique stationary distributions $P_{0}$ and $P_{s t}$ exist for the dynamics defined by $\mathcal{M}_{0}$ and $\mathcal{M}$, respectively, i.e.,

$$
\begin{aligned}
& \mathcal{M} P_{0}=0, \\
& \mathcal{M} P_{\text {st }}=0,
\end{aligned}
$$

Eqs. (A4) and (A6) lead to

$$
P_{s t}=\lim _{t \rightarrow \infty}\left[U(t) P_{0}\right]=P_{0}+\int_{0}^{\infty} \mathrm{d} t U(t) \mathcal{M} P_{0} .
$$

The arbitrariness in the splitting of $\mathcal{M}$ allows one to choose $P_{0}$ as the local equilibrium distribution

$$
P_{0}=P_{e q}^{C} \exp \left[-\sum_{x=1}^{L} \mu(x) n(x)\right] .
$$

For $\delta_{\mu} \ll 1$ one has $\mu(x) \simeq \mu+\delta_{\mu}(x / L)$; applying $\mathcal{M}$ to $P_{0}$ [see Eq. (A1)] and by using the detailed balance condition one obtains

$$
\begin{aligned}
\mathcal{M} P_{0}=\sum_{x=1}^{L-1} & \left\{u\left(n_{x}, n_{x+1}\right)\left[\mathrm{e}^{-\frac{\delta_{\mu}}{L}}-1\right]\right. \\
& \left.+u\left(n_{x+1}, n_{x}\right)\left[\mathrm{e}^{\frac{\delta_{\mu}}{L}}-1\right]\right\} P_{0}\left(\left\{n_{1}, n_{L}\right\}\right) .
\end{aligned}
$$

The aim is to calculate the mean current $\langle j\rangle_{s t}$, from which one can obtain $D$ in the limit of small density differences by using Eq. (35). Averaging $j$, given by Eq. (42), over the distribution $P_{s t}$ and expanding to first order in $\delta$ leads to

$$
\begin{aligned}
\left\langle j_{x+1}\right\rangle_{s t}=\frac{\delta_{\mu}}{L}\left[\left\langle u\left(n_{x}, n_{x+1}\right)\right\rangle_{0}\right. & \\
& \left.-\sum_{x^{\prime}=1}^{L} \int_{0}^{\infty} \mathrm{d} t^{\prime}\left\langle j_{x+1} U\left(t^{\prime}\right) j_{x^{\prime}}\right\rangle_{0}\right]+O\left(\frac{\delta_{\mu}^{2}}{L^{2}}\right) .
\end{aligned}
$$

where $\langle\cdot\rangle_{0}$ indicate the average taken over the local equilibrium distribution [Eq. (A8)]. By taking the limits 
$\delta_{\mu} \rightarrow 0$ and $L \rightarrow \infty$ we obtain the Green-Kubo formula

$$
\begin{aligned}
D(\rho)=\frac{1}{\chi} & {\left[\left\langle u\left(n_{x}, n_{x+1}\right)\right\rangle_{e q}\right.} \\
& \left.-\sum_{x^{\prime}=1}^{\infty} \int_{0}^{\infty} \mathrm{d} t^{\prime}\left\langle j_{x+1} U_{e q}\left(t^{\prime}\right) j_{x^{\prime}}\right\rangle_{e q}\right]
\end{aligned}
$$

with $\chi=\left\langle n_{x}^{2}\right\rangle_{e q}-\left\langle n_{x}\right\rangle_{e q}^{2}$. The limits $\delta_{\mu} \rightarrow 0$ and $L \rightarrow \infty$ imply that $P_{0} \rightarrow P_{e q}$ and $U_{0} \rightarrow U_{e q}=\mathrm{e}^{\mathcal{M}_{e q} t}$. Note that in Eq. A11 the averages are taken over the equilibrium distribution and the properties of the diffusion coefficient are completely determined by the equilibrium dynamics of the model. Accordingly, the r.h.s. of Eq. A11 is independent of $x$ and thus $D$ depends on $\rho$ only.

\section{APPENDIX B: INFLUENCE OF THE RESERVOIR DENSITIES ON THE DENSITY PROFILES}

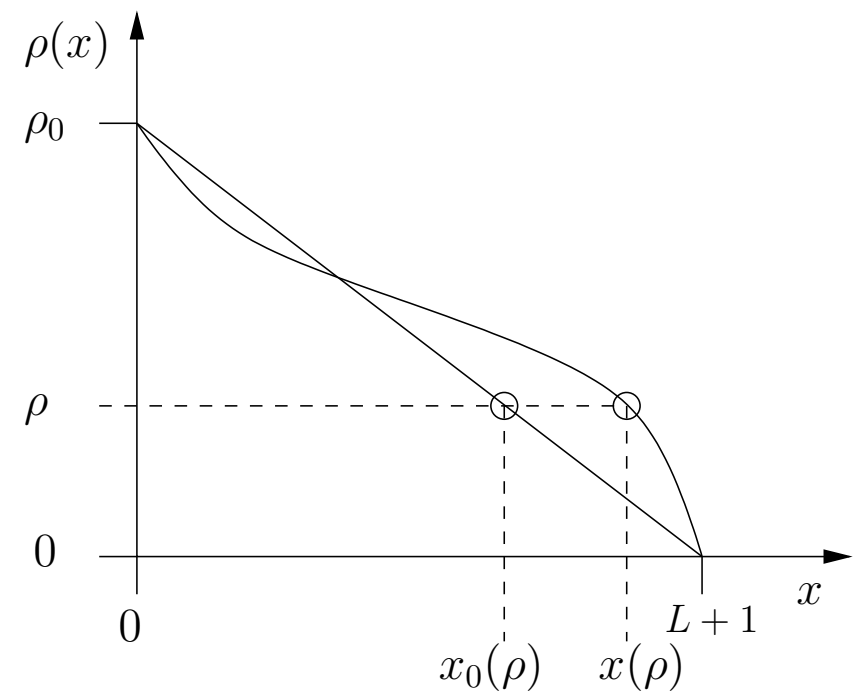

FIG. 8: Schematic comparison between a typical steady-state density profile (curved line) and free diffusion (straight line). At a given density $\rho$ the corresponding positions $x(\rho)$ and $x_{0}(\rho)$ are taken from the steady-state density profile and from the free-diffusion line, respectively, and $\Delta x(\rho)$ is determined from Eq. (B1).

In Sec. IV] we have shown that, within our model, the density-dependent diffusion coefficient $D(\rho)$ does not depend on the boundary conditions, i.e., the densities of the left and right reservoirs. As shown in Fig. 5, the density profiles for low reservoir densities seemingly lie mostly above the free diffusion straight line [see Fig. 5(b)], whereas the opposite happens for high reservoir densities [see Fig. 55(a)]. While these profiles refer to different interaction strength $w$, we note that the difference between $D(\rho)$ for $w=2$ and for $w=1.5$ [see Figs. 6)(b) and $7(\mathrm{a})$ ] is not very marked. Therefore it is unlikely that this difference explains the differences between the profile corresponding to $w=1.5$ in Fig. 5(a) and the profile corresponding to $w=2$ in Fig. 5(b).

In order to clarify the relation between the steady-state profile and the boundary conditions, here we restrict our analysis to the case in which the right reservoir is a perfect sink, $\left(\rho_{L+1}=0\right)$ and the left reservoir density $\rho_{0}$ is varied, keeping the interaction strength $w$ constant. The definitions in Eq. (21) for the rates at the boundaries have been used, so that the density profile is a continuous function of $x$.

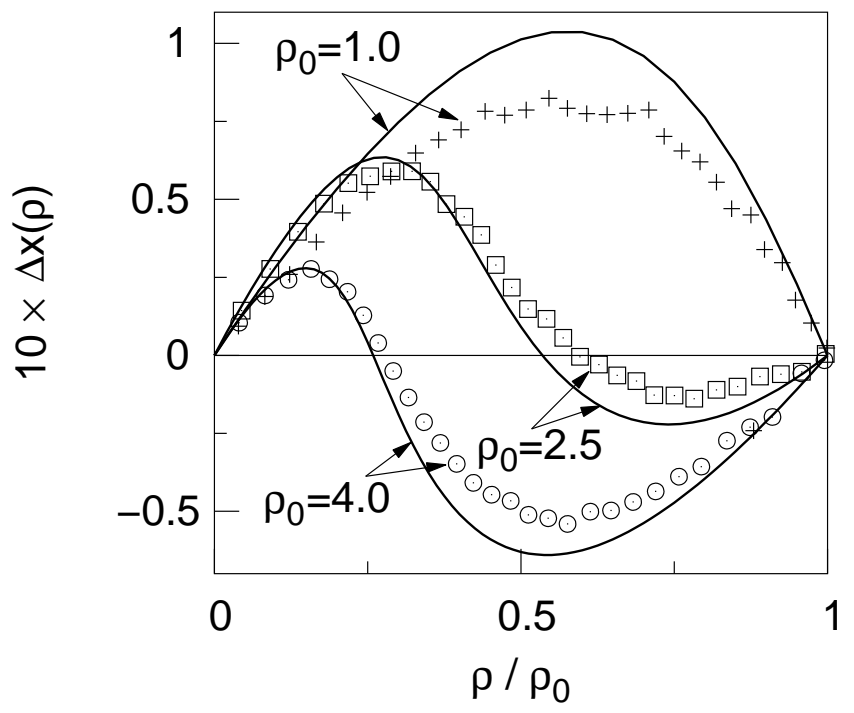

FIG. 9: $\Delta x(\rho)$ [see Eq. (B1)] for left reservoir densities $\rho_{0}=1$ $(+), \rho_{0}=2.5(\bullet)$ and $\rho_{0}=4(\odot)$ with fixed $w=1.5, L=300$, and $\rho_{L+1}=0$. The full lines correspond to $\Delta x(\rho)$ obtained by using the mean-field diffusion coefficient [Eq. [56]. The quantity $\Delta x(\rho)$ in the $y$-axis is multiplied by a factor 10 in order to enhance visibility.

A convenient quantity to describe the dependence on $\rho_{0}$ of the profiles is the deviation in $x$ at fixed $\rho$ between the actual density profile and the straight line corresponding to free diffusion (see Fig. 8), defined as

$$
\Delta x(\rho)=\frac{x(\rho)-x_{0}(\rho)}{L+1},
$$

where $x(\rho)$ is the inverted steady-state profile and $x_{0}(\rho)=(L+1)\left(1-\rho / \rho_{0}\right)$ is the corresponding free diffusion line. In Fig. 9] we report the numerical data for $\Delta x(\rho)$ corresponding to three left reservoir densities $\left(\rho_{0}=1,2.5\right.$, and 4$)$. These results exhibit the typical behavior of the deviation $\Delta x$ : at small $\rho_{0}$ it is positive for all $\rho\left(\rho_{0}=1\right)$ whereas upon increasing the reservoir density a negative part appears $\left(\rho_{0}=2.5\right.$ and 4$)$. This pattern can be explained along the line of arguments introduced in Sec. IV] based on the coarse-grained description of the diffusion-like behavior. The nonlinear diffusion equation 
[Eq. (30)] yields for the inverse steady-state profile $x(\rho)$

$$
\frac{\mathrm{d} x(\rho)}{\mathrm{d} \rho}=-\frac{D(\rho)}{j},
$$

where $j$ is the current and $D$ the diffusion coefficient. Integrating Eq. (B2) one obtains

$$
x(\rho)=\frac{1}{j} \int_{\rho}^{\rho_{0}} \mathrm{~d} \xi D(\xi),
$$

where $0 \leq \rho \leq \rho_{0}$. Imposing the boundary condition $x(0)=L+1$ at the right end of the system leads to

$$
j=\frac{1}{L+1} \int_{0}^{\rho_{0}} \mathrm{~d} \xi D(\xi),
$$

so that $x(\rho)$ is given by

$$
x(\rho)=(L+1)\left[\int_{0}^{\rho_{0}} \mathrm{~d} \xi D(\xi)\right]^{-1} \int_{\rho}^{\rho_{0}} \mathrm{~d} \xi D(\xi) .
$$

By combining Eqs. (B1) and (B5) one obtains

$$
\begin{aligned}
\Delta x(\rho) & =\frac{x(\rho)-x_{0}(\rho)}{L+1}= \\
& =\left[\int_{0}^{\rho_{0}} \mathrm{~d} \xi D(\xi)\right]^{-1} \int_{\rho}^{\rho_{0}} \mathrm{~d} \xi D(\xi)-\frac{\rho_{0}-\rho}{\rho_{0}},
\end{aligned}
$$

which, as expected, vanishes for $\rho=0$ and $\rho=\rho_{0}$. One can re-write the last equation as follows:

$$
\begin{aligned}
\Delta x(\rho)=\rho & {\left[\int_{0}^{\rho_{0}} D(\xi) \mathrm{d} \xi\right]^{-1} } \\
& \times\left[\frac{1}{\rho_{0}} \int_{0}^{\rho_{0}} \mathrm{~d} \xi D(\xi)-\frac{1}{\rho} \int_{0}^{\rho} \mathrm{d} \xi D(\xi)\right] .
\end{aligned}
$$

Note that $\rho\left[\int_{0}^{\rho_{0}} D(\xi) \mathrm{d} \xi\right]^{-1}$ is nonnegative whereas the second factor on the r.h.s. of Eq. (B7) can change sign. Denoting this latter factor by $h(\rho)$ and introducing the function $f(\rho)=D(\rho)-1$, one obtains

$$
h(\rho)=\frac{1}{\rho_{0}} \int_{0}^{\rho_{0}} \mathrm{~d} \xi f(\xi)-\frac{1}{\rho} \int_{0}^{\rho} \mathrm{d} \xi f(\xi)=R\left(\rho_{0}\right)-R(\rho),
$$

where $R(\rho)=\frac{1}{\rho} \int_{0}^{\rho} \mathrm{d} \xi[D(\xi)-1]$. Within our model the function $f$ is always positive (apart from Fig. 6(a) for $\rho \rightarrow 0$ and some noisy data at large $\rho$ in Figs. 6 and 7) and it vanishes for $\rho \rightarrow 0$ and $\rho \rightarrow \infty$. Thus $R(\rho)$ also vanishes for $\rho \rightarrow 0$ or $\rho \rightarrow \infty$ and hence has a maximum at a certain $\rho_{M}$, with $0<\rho_{M}<\infty$. A sketch of this function is provided in Fig. 10. Two qualitatively different behaviors emerge by varying the left reservoir density $\rho_{0}$. If the reservoir density is set to $\rho_{0}^{<} \leq \rho_{M}$, then in the interval $0 \leq \rho \leq \rho_{0}^{<}$no solution to $R\left(\rho_{0}^{<}\right)-R(\rho)=0$ can be found other than the trivial one $\rho=\rho_{0}^{<}$. In this situation, we have $R(\rho)<R\left(\rho_{0}^{<}\right)$so that the function $\Delta x(\rho)$ is always positive in the considered interval of densities,

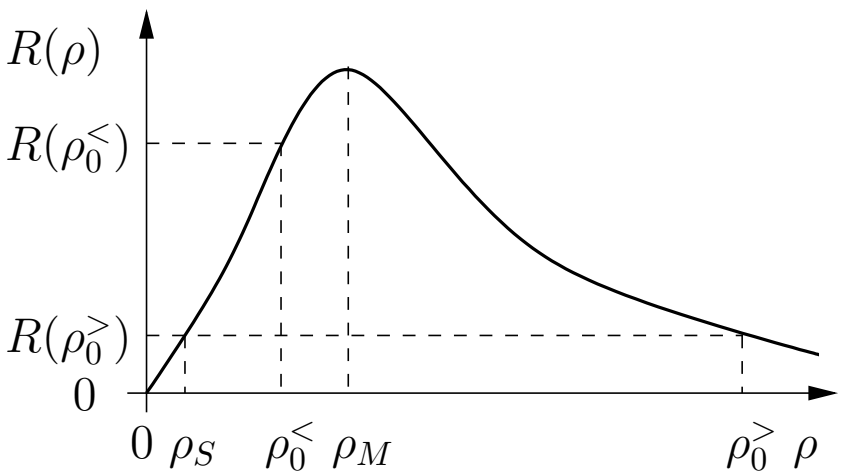

FIG. 10: Qualitative plot of the function $R(\rho)$ (see Eq. (B8)). Two possible situations are shown: if the reservoir density $\rho_{0}^{<}$is smaller than $\rho_{M}$, there are no densities $0 \leq \rho<\rho_{0}^{<}$ such that $R(\rho)=R\left(\rho_{0}^{<}\right)$. In the opposite case with reservoir density $\rho_{0}^{>}>\rho_{M}$, there exist a density $0<\rho_{S}<\rho_{0}^{>}$such that $R\left(\rho_{S}\right)=R\left(\rho_{0}^{>}\right)$.

and the density profile always lies above the line corresponding to free diffusion. This is the case for $\rho_{0}=1$ and substrate interaction $w=1.5$ as shown in Fig. 9. In the opposite situation, in which the density of the reservoir is set to $\rho_{0}^{>} \geq \rho_{M}$, there always exists a density $\rho_{S}<\rho_{0}^{>}$ such that $R\left(\rho_{0}^{>}\right)-R\left(\rho_{S}\right)=0$. At this density the density profile crosses the free diffusion line. For $\rho>\rho_{S}$, $\Delta x(\rho)>0$ while $\Delta x(\rho)<0$ for $\rho<\rho_{S}$. In Fig. 9 this corresponds to the cases $\rho_{0}=2.5$ and $\rho_{0}=4$.
[1] N. Giordano and J.T. Cheng, J. Phys: Condens. Matter 13, R271 (2001).

[2] P.R. Selvaganapathy, E.T. Carlen, and C.H. Mastrangelo, Proceedings of the IEEE 91, 954 (2003).

[3] R. Mukhopadhyay, Analy. Chem. 78, 7379 (2006).

[4] J.L. Pearson and D.R.S. Cumming, Micro. Eng. 78-79, 343 (2005).

[5] D. Mijatovich, J.C.T. Eijkel, and A. van den Berg, Lab on a Chip 5, 492 (2004).

[6] S. Supple and N. Quirke, Phys. Rev. Lett. 90, 214501
(2003).

[7] G. Hummer, J.C. Rasaiah, and J.P. Noworyta, Nature 414, 188 (2001).

[8] S. Dietrich, M.N. Popescu, and M. Rauscher, J. Phys: Condens. Matter 17, S577 (2005).

[9] J.A. Nieminen, D.B. Abraham, M. Karttunen, and K. Kaski, Phys. Rev. Lett. 69, 124 (1992).

[10] G. Oshanin, J. De Coninck, A.M. Cazabat, and M. Moreau, Phys. Rev. E 58, R20 (1998).

[11] M. Vouè and J. De Coninck, Acta Mater. 48, 4405 (2000). 
[12] J. Koplik, T.S. Lo, M. Rauscher, and S. Dietrich, Phys. Fluids 3, 032104 (2006).

[13] F. Heslot, A.M. Cazabat, and N. Fraysse, J. Phys.: Condens. Matter 1, 5794 (1989).

[14] F. Heslot, A.M. Cazabat, and P. Levinson, Phys. Rev. Lett. 62, 1286 (1989).

[15] F. Heslot, A.M. Cazabat, P. Levinson, and N. Fraysse, Phys. Rev. Lett. 65, 599 (1990).

[16] J. Daillant, J.J. Benattar, and L. Leger, Phys. Rev. A 41, 1963 (1990).

[17] U. Albrecht, A. Otto, and P. Leiderer, Phys. Rev. Lett. 68, 3192 (1992).

[18] N. Fraysse, M.P. Valignant, F. Heslot, A. M. Cazabat, and P. Levinson, J. Colloid Interface Sci. 158, 27 (1993).

[19] M. Vouè, M.P. Valignant, G. Oshanin, A. M. Cazabat, and J. De Coninck, Langmuir 14, 5951 (1998).

[20] E. Pérez, E. Schäffer, and U. Steiner, J. Colloid Interface Sci 234, 178 (2001).

[21] S. F. Burlatsky, G. Oshanin, A. M. Cazabat, and M. Moreau, Phys. Rev. Lett. 76, 86 (1996).

[22] G. Oshanin, J. De Coninck, A.M. Cazabat, and M. Moreau, J. Mol. Liq. 76, 195 (1998).

[23] M.N. Popescu and S. Dietrich, Phys. Rev. E 69, 061602 (2004).

[24] M.N. Popescu, S. Dietrich, and G. Oshanin, J. Phys: Condens. Matter 17, S4189 (2005).

[25] T. Chou, Phys. Rev. Lett. 80, 85 (1998).

[26] T. Chou, J. Chem. Phys. 110, 606 (1999).

[27] T. Chou, Biophys. J. 86, 2827 (2004).

[28] T. Chou, J. Phys.: Math. Gen. A 39, 2253 (2006).
[29] K. K. Mon and J. K. Percus, J. Chem. Phys. 119, 3343 (2003).

[30] S. K. Bhatia, O. Jepps, and D. Nicholson, J. Chem. Phys. 120, 4472 (2004).

[31] P. Demontis, G. Stara, and G. Suffritti, J. Chem. Phys. 120, 9233 (2004).

[32] F. Marchesoni and A. Taloni, Phys. Rev. Lett. 97, 106101 (2006).

[33] C. Lutz, M. Kollmann, P. Leiderer, and C. Bechinger, J. Phys.: Condens. Matter 16, S4075 (2004).

[34] S. M. Saparov, J. R. Pfeifer, L. Al-Momani, G. Portella, B. L. de Groot, U. Koert, and P. Pohl, Phys. Rev. Lett. 96, 148101 (2006).

[35] E. Andjel, C. Cocozza-Thivent, and M. Roussignol, Ann. de l'I.Henry Poincaré 21, 363 (1985).

[36] E. Andjel, C. Cocozza-Thivent, and M. Roussignol, Z. f. Wahrscheinlichkeitstheorie 70, 509 (1985).

[37] C. Godrèche, preprint cond-mat/0604276 (2006).

[38] C. Godrèche and J. M. Luck, Eur. Phys. J. B 21, 473 (2001).

[39] K. Binder, in Monte Carlo Methods in Statistical Physics, edited by K. Binder (Springer, Berlin, 1986), p. 30.

[40] A.B. Bortz, M.H. Kalos, and J.L. Lebowitz, J. Comput. Phys. 17, 10 (1975).

[41] L. Bertini, A. da Sole, D. Gabrielli, G. Jona-Lasinio, and C. Landim, J. Stat. Phys. 107, 635 (2002).

[42] H. Spohn, Large scale dynamics of interacting particles (Springer, Berlin, 1991). 